INTER NATIONAL MONETARY FUND
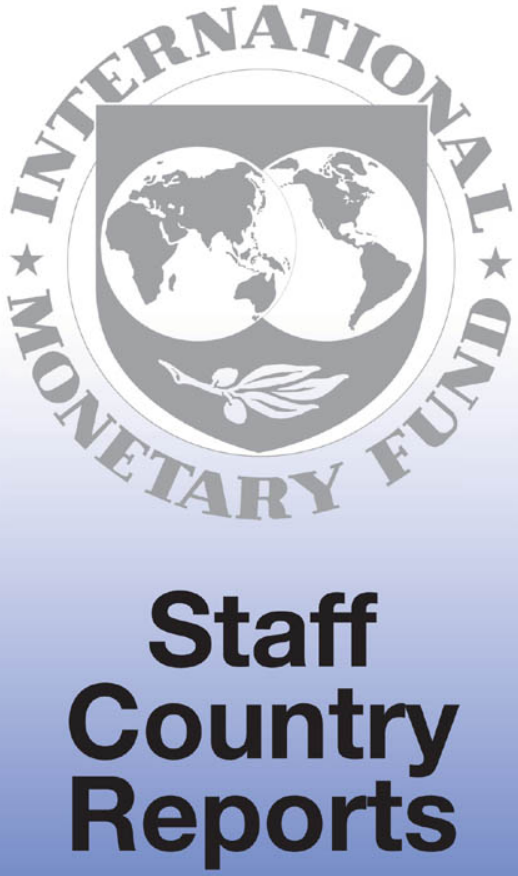


\section{Cape Verde: First Review Under the Policy Support Instrument and Requests for Waivers of Nonobservance and Modification of Assessment Criteria-Staff Report; Supplement; and Press Release}

In the context of the first review under the Policy Support Instrument and requests for waivers of nonobservance and modification of assessment criteria with the Republic of Cape Verde, the following documents have been released and are included in this package:

- $\quad$ The staff report for the first review under the Policy Support Instrument and requests for waivers of nonobservance and modification of assessment criteria, prepared by a staff team of the IMF, following discussions that ended on May 31, 2011 with the officials of Cape Verde on economic developments and policies. Based on information available at the time of these discussions, the staff report was completed on July 7, 2011. The views expressed in the staff report are those of the staff team and do not necessarily reflect the views of the Executive Board of the IMF.

- $\quad$ A supplement to the staff report

- A press release summarizing the views of the Executive Board.

The documents listed below have been or will be separately released.

- Letter of Intent sent to the IMF by the authorities of Cape Verde*

- Memorandum of Economic and Financial Policies by the authorities of Cape Verde*

- Technical Memorandum of Understanding*

*Also included in Staff Report

The policy of publication of staff reports and other documents allows for the deletion of market-sensitive information.

Copies of this report are available to the public from

International Monetary Fund $\bullet$ Publication Services

$70019^{\text {th }}$ Street, N.W. $\bullet$ Washington, D.C. 20431

Telephone: (202) 623-7430 • Telefax: (202) 623-7201

E-mail: publications@imf.org Internet: http://www.imf.org

\section{International Monetary Fund Washington, D.C.}




\title{
CAPE VERDE
}

\section{First Review Under the Policy Support Instrument and Requests for Waivers of Nonobservance and Modification of Assessment Criteria}

\author{
Prepared by the African Department \\ (In consultation with other departments) \\ Approved by Sharmini Coorey and Claire Waysand
}

July 7, 2011

\section{Executive Summary}

Focus: The first review under the policy support instrument (PSI) focused on policies to guide the country's continued recovery from the global economic crisis and to rebuild fiscal and reserve buffers. Economic recovery in Cape Verde continues unabated, driven by the tourism sector and a rapid implementation of the public investment program. Authorities remain committed to macroeconomic stability.

Assessment: All but two end-March 2011 assessment criteria (AC) were met, and progress has been made on the structural benchmarks. Additionally, all but one end-December 2010 indicative targets have been met. The missed target and ACs reflect temporary and minor factors, and the program remains on track. Staff recommends completing the first review.

\section{Policy discussions:}

- $\quad$ Staff and the authorities agree that the public investment program will help relieve infrastructure bottlenecks, but the fiscal deficit must be scaled back in the next few years to reduce the external debt ratio after 2012 and to maintain debt sustainability.

- $\quad$ The Bank of Cape Verde (BCV) has maintained a tight stance in the face of ongoing external shocks and volatile flows, but it should relax monetary policy stance as reserves accumulate. The BCV is keen to enhance liquidity management and improve the efficiency of the financial system.

- $\quad$ A policy mix of an expansionary fiscal policy and a tight monetary policy creates medium-run macroeconomic risks.

- Staff encouraged the authorities' structural reform intentions in the following areas: (i) reform the state-owned enterprises to improve management incentives and internal efficiency; (ii) strengthen banking sector regulation and supervision (e.g., new banking law); and (iii) improve the flexibility of the labor market.

Exchange rate regime: Conventional peg to the euro.

\footnotetext{
${ }^{1}$ Discussions took place during May 18-31, 2011. The team comprised Valerie Cerra (head), Yibin Mu, Fabiano Rodrigues Bastos, and Abdullah Al-Hassan (all AFR). The mission met with Finance Minister Cristina Duarte, Central Bank Governor Carlos Burgo, Parliamentarians and other government officials, and representatives of civil society and the private sector. Daouda Sembene (OED) participated in the discussions.
} 
I. Background and PSI performance.....

II. Policy discussions

A. Fiscal policy: Balancing Investment-Led Growth with Debt Sustainability ............ $\underline{6}$

B. Monetary Policy: A Restrictive Stance to Support the Exchange Rate Peg

C. Macroeconomic Policy Mix: Strengthening Coordination

D. Financial Sector Strategy: Enhancing the Regulatory Framework

E. Revitalizing State Owned Enterprises

F. Improving Labor Market Flexibility

III. Program Monitoring.

IV. Staff Appraisal

\section{Boxes}

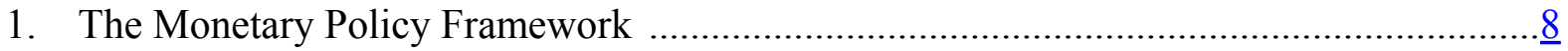

2. Labor Markets in Cape Verde......................................................................... 10

Figures

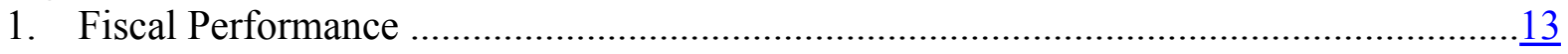

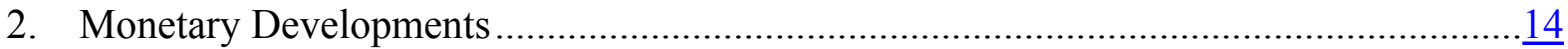

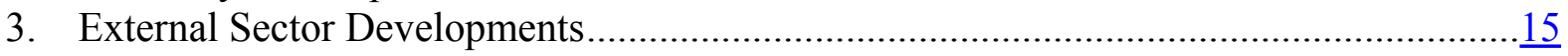

Tables

1. Selected Economic Indicators, 2009-15....

2. Fiscal Operations of the Central Government, 2009-15 (millions of escudos)...............17

3. Fiscal Operations of the Central Government, 2009 (percent of GDP)..........................18

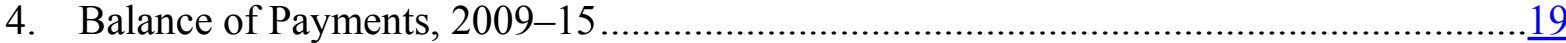

5. Monetary Survey, 2009-15 ..........................................................................

6. Central Bank Survey, 2009-15 ......................................................................

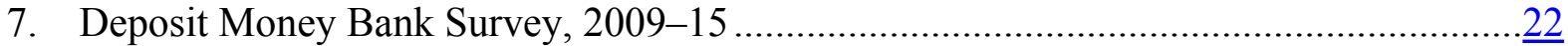

8. Financial Soundness of the Banking Sector............................................................ 23

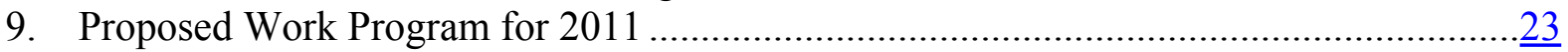

10. Quantitative Assessment Criteria and Indicative Targets for 2010-11 under the PSI .....24

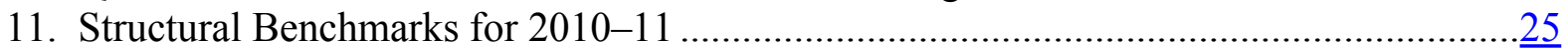

Appendix

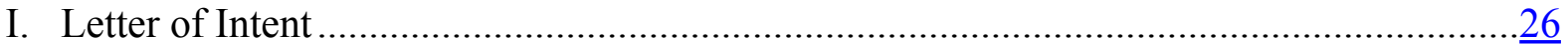

Attachment I. Memorandum of Economic and Financial Policies ...................................28

Attachment II. Technical Memorandum of Understanding

Annexes

I. Relations with the Fund

II. Joint IMF-World Bank Management Action Plan-Implementation Matrix ................... $\underline{44}$

III. Statistical Issues 


\section{BACKGRound AND PSI PERformanCe}

\section{A. Recent Developments and Outlook}

1. The economic recovery is firmly under way. Real GDP grew by 5.4 percent in 2010 , backed by strong growth in the number of tourists, lured by lower prices. Confidence indicators herald an improved economic outlook this year, and staff projects growth to edge up to 5.6 percent. Net exports should contribute to growth with continued strong performance in the tourism sector and fisheries. Implementation of the public investment program (PIP) should support domestic demand, as corroborated by high growth in imports of consumer and intermediate goods during the first quarter of 2011.
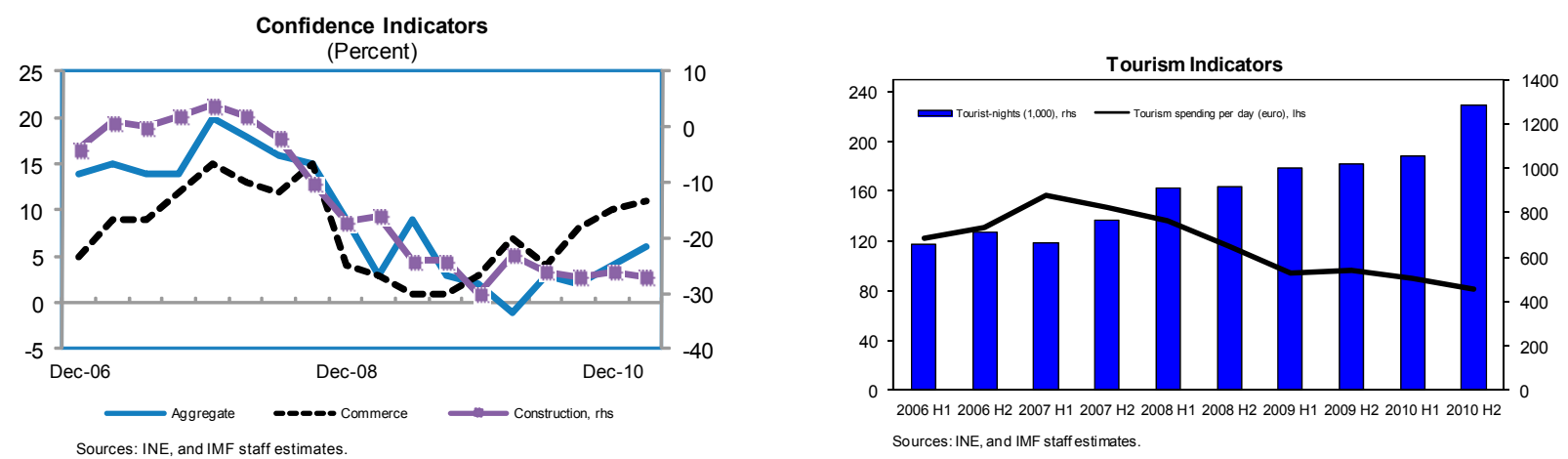

2. Inflation rose in recent months due to hikes in global oil and food prices.

Consumer price inflation surged to 5.3 percent in April 2011 (year-on-year), driven by food and utility prices. The government eliminated subsidies for water and electricity, allowing full pass-through of prices. Core inflation, which excludes food and energy, remains subdued.
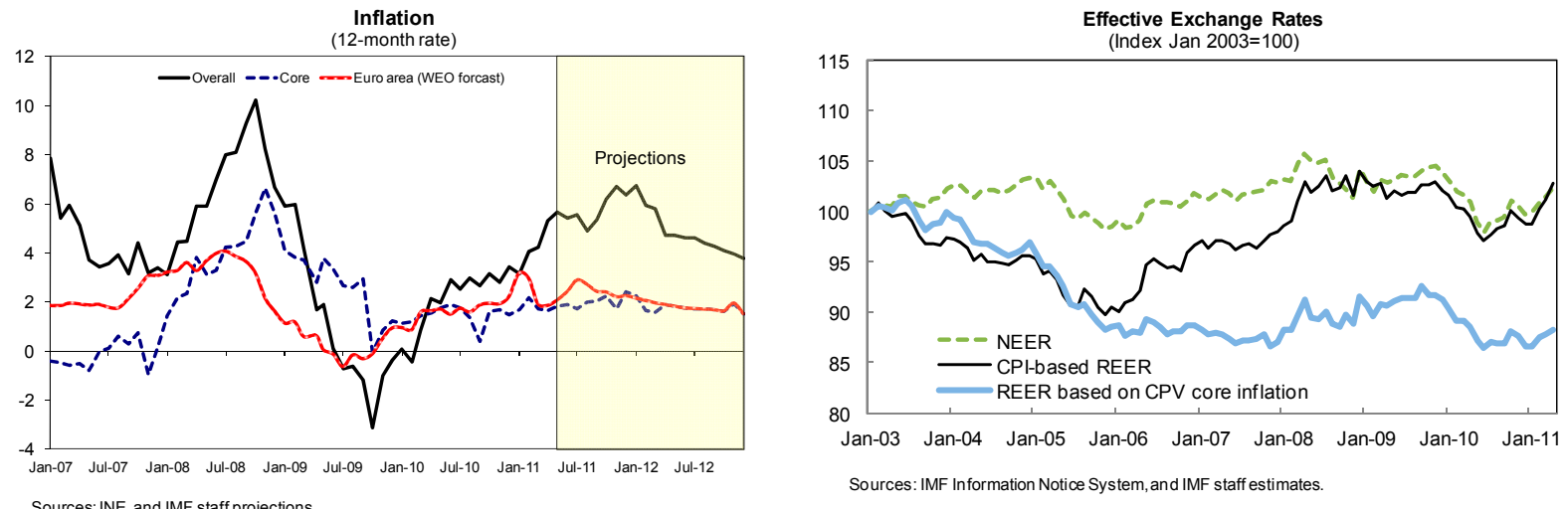

3. Fiscal performance in $\mathbf{2 0 1 0}$ was better than projected. The fiscal deficit was 10.7 percent of GDP, lower than the 13.5 percent deficit projected at the time of the last staff report (November 2010). Capital expenditures were around 15 percent below forecast due to 
delays in project implementation. The government also constrained recurrent expenditures in response to weaker revenue outturns. Net domestic borrowing remained low. ${ }^{2}$

\section{The current account deficit declined modestly and reserves continued to rise in}

2010. The current account deficit was 11.3 percent of GDP, reflecting the slower-thanexpected (albeit strong) PIP implementation, which resulted in lower growth in capital imports compared to previous projections. ${ }^{3}$

The level of gross international reserves increased by nearly 20 million euros, mainly driven by a significantly higher level of official disbursements in 2010 compared to 2009 as the authorities took advantage of the external concessional financing made available to the country during its transition from least

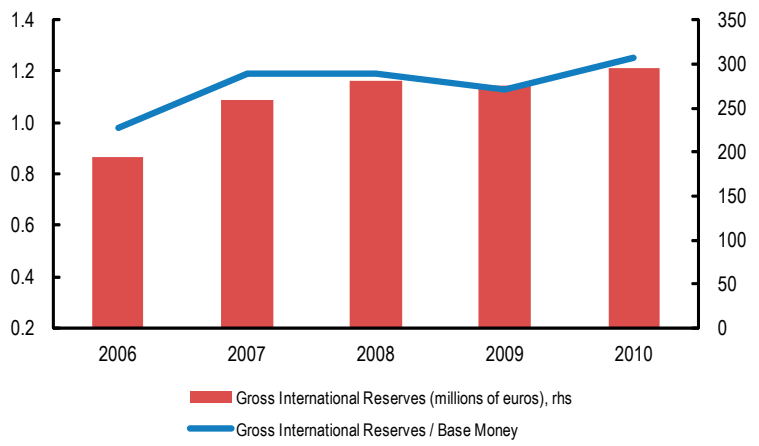
developed to middle-income country status. ${ }^{4}$
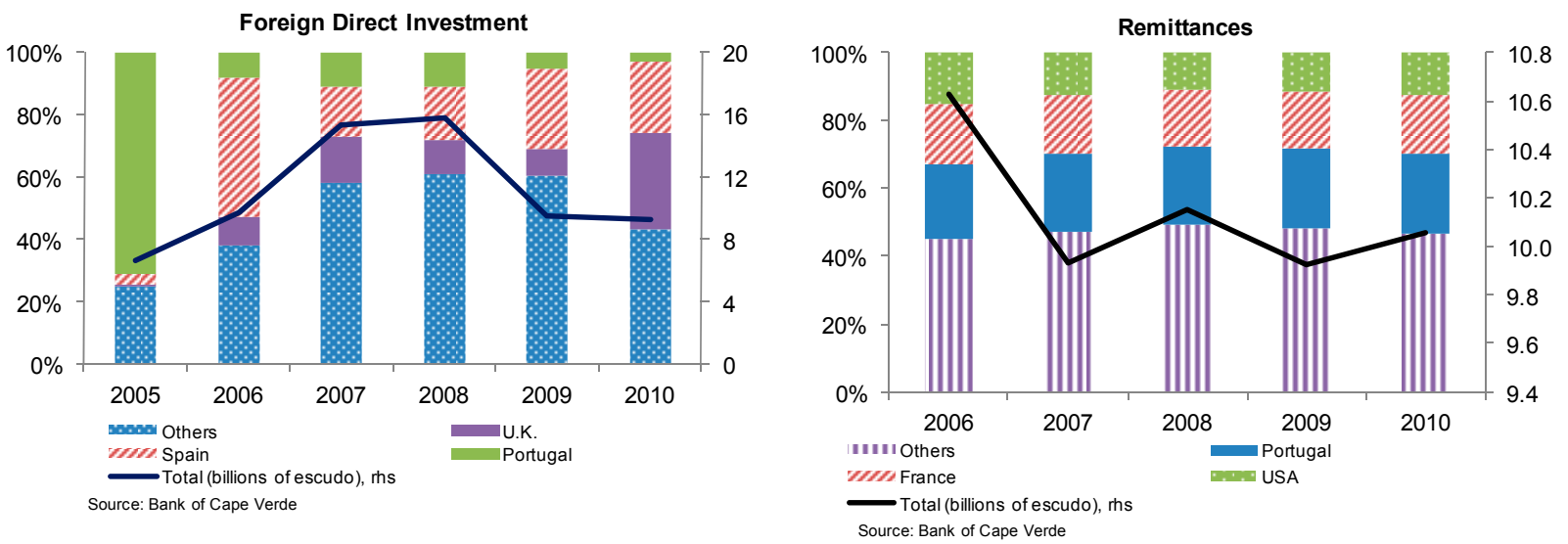

\footnotetext{
${ }^{2}$ The downward revision of the stock of public external debt at end-2010 reflects valuation effects and lower-thanprojected disbursements.

${ }^{3}$ The lower current account outturn relative to the projection in November 2010 staff report (18 percent of GDP) also reflects negative errors and omissions ( 3 percent of GDP) in the official outturn data, which likely includes some current account components.

${ }^{4}$ With Cape Verde's graduation from the least-developed-country status, donors have provided a transitional period for concessional funding over several years. The authorities are using this window of opportunity to finance as much as possible of the infrastructure investment needs on favorable terms. The World Bank and outside consultants have appraised large projects.
} 
5. However, gross reserves declined by around 23 million euros in the first quarter of 2011. Reserve losses reflected the paucity of official loan disbursements, higher import prices, a negative outflow of trade credits, accelerated amortization of the private external debt, and increased repatriation of dividends to foreign investors.

6. The BCV has maintained its tight monetary stance. The BCV has kept its policy rate at 4.25 percent since January 2010, representing a large spread to the euribor, even adjusting for a risk premium. Private sector credit and emigrant deposits grew at 8.9 percent and 6.6 percent, respectively, in 2010.

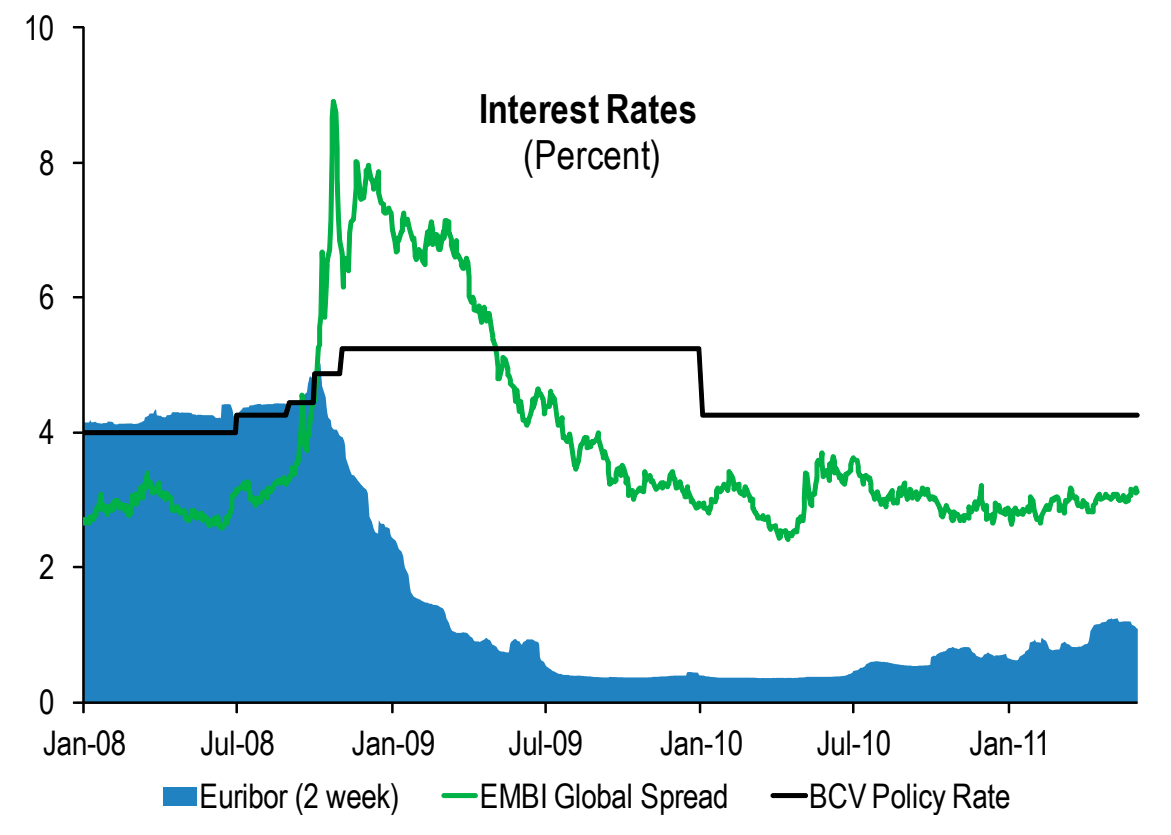

Sources: Bank of Cape Verde, Thompson Datastream, Bloomberg.

7. Risks to the outlook remain balanced. Medium-term prospects are favorable, with Cape Verde's large fiscal and current account deficits financed mainly by concessional flows. The PIP should relieve infrastructure bottlenecks, which should underpin growth in foreign direct investment (FDI) and exports. Cape Verde has strong linkages with North-America and Europe, and recent turbulence in the euro zone poses the greatest risk to tourism, FDI, and remittances. So far, trade and financial flows have remained buoyant despite external turmoil, and development partners have expressed general willingness to maintain existing credit lines. Meanwhile, political uncertainty in the Middle East and North African (MENA) region has diverted some tourists to Cape Verde. The negative terms of trade shock, currently stoking inflation and weakening the external position, should ease in late 2011, according to WEO assumptions.

\section{B. Program Performance}

8. All but one end-December 2010 indicative targets and all but two end-March 2011 assessment criteria were met (MEFP, Table 1). 
- A minor nonobservance of the end-December indicative target on net domestic borrowing of the central government was more than offset by significant overperformance on the end-March assessment criterion.

- $\quad$ Net domestic assets of the central bank accumulated beyond the end-March 2011 ceiling (assessment criterion) due to a technical timing issue that reflects a temporary factor: the government received large disbursements in late 2010 and wrote checks, which cleared in the first quarter of 2011, running down government deposits.

- $\quad$ The BCV significantly over-performed its end-December 2010 indicative target on the accumulation of net international reserves, but missed the end-March assessment criterion. In particular, the minor nonobservance of the AC reflects weak first quarter private balance of payments flows, including an unusually large outflow of trade credits, which tend to be volatile. The authorities and staff expect the balance of payments to improve in the second half of 2011, particularly given large new FDI projects coming on stream and the opening of new hotels.

\section{Three structural benchmarks have been missed, but the government remains committed to all measures (MEFP, Table 2).}

- $\quad$ The BCV and the Ministry of Finance are working to finalize the fiscal-monetary coordination agreement and the memorandum of understanding for the financial stability committee. There is high-level commitment to address lingering issues in the coming months.

- $\quad$ Issuance of fungible treasury bonds was delayed until June 2011 when the new IT platform for auctioning treasury securities will be launched.

- $\quad$ The medium-term strategy on debt should be ready in September 2011, after technical assistance by the World Bank and IMF in July 2011.

\section{Policy Discussions}

\section{A. Fiscal Policy: Balancing Investment-Led Growth with Debt Sustainability}

10. The fiscal policy strategy emphasizes efficient implementation of the PIP while safeguarding debt sustainability. Infrastructure gaps and unreliable provision of electricity and water limit private sector expansion and drive up costs. Staff continues to support the scaled-up PIP, given the country's development needs and the predominant reliance on concessional external financing. The authorities are keen to enhance project evaluation and control, and are committed to improving capacity in debt management and sustainability analysis with the support of the World Bank and the IMF (MEFP $\$ 10$ ). Indeed, the 2011 budget and 2012-14 MTFF project a declining fiscal deficit, with a capital spending 
envelope similar to previous plans. However, donors recently agreed to consider an extension of the concessional window until 2015 and the government is contemplating new investment projects. ${ }^{5}$ The mission stressed that the focus should be on implementing projects already in the pipeline and new commitments should be avoided so as to reduce debt ratios over the medium run. Moreover, staff emphasized that any large new projects should have incontrovertible social benefits or facilitate export expansion, and should be consistent with debt sustainability. They should also undergo a feasibility study even if funded with concessional loans. Risk- and cost-sharing arrangements with the private sector should also be carefully assessed.

\section{The government demonstrated firm resolve to rein in current spending. The} authorities eliminated subsidies for electricity and water, and allowed domestic gas prices to adjust to international prices. ${ }^{6}$ They submitted a budget proposal to Parliament that includes a freeze of public sector wages this year. These measures are contributing to a lower projected deficit for 2011 than previously expected. The authorities were adamant about their continued ability to control costs and keep domestic debt low, while also reorienting budget resources to support vulnerable groups if necessary (MEFP $\mid 9$ ).

12. The authorities intend to improve revenue generation. The authorities are confident of achieving their optimistic revenue projections this year. Revenues are expected to grow faster than nominal GDP, partly reflecting higher collection of tax arrears as the economy recovers. Still, the government acknowledges the need to strengthen tax administration and has requested technical assistance from the IMF. More broadly, the authorities are committed to a thorough tax reform, including simplifying the tax system and rationalizing tax exemptions (MEFP q8), which staff supports.

\section{B. Monetary Policy: A Restrictive Stance to Support the Exchange Rate Peg}

\section{The BCV is prepared to tighten monetary policy further if needed to support}

reserves. Although the global crisis has subsided, the BCV sees continued external risks, particularly in their main trading partners in the euro area. The weak first quarter balance of payments highlighted such concerns. Nonetheless, the Treasury is confident that disbursements of official loans will pick up in the second half of the year, supporting foreign exchange inflows. Notwithstanding staff's advice to BCV to investigate the outflow of trade credits and closely monitor the level of reserves, staff considers the outlook for the balance of

\footnotetext{
${ }^{5}$ As a hypothetical scenario, if Cape Verde would decide to maintain the level of foreign-financed capital expenditure planned for 2012 unchanged over the following 3 years, the external debt as a percentage of GDP in 2015 would climb up to approximately 63.5 instead of the 55.3 currently projected. This would significantly increase fiscal vulnerabilities while stretching absorptive capacity as there would be fewer opportunities for high return public investments.

${ }^{6}$ The authorities estimate the fiscal savings from those measures could reach 0.3 percent of GDP.
} 
payments to be favorable and reserve coverage to be adequate. Staff therefore sees space to relax monetary policy as reserves accumulate.

\section{The BCV is committed to improving its liquidity management and monetary} policy operations. The BCV recently changed its auction procedure to reduce structural liquidity and encourage interbank market development, and plans to improve daily liquidity management (MEFP -13). Staff encouraged the BCV to undertake other reforms suggested by the FSAP and MCM TA missions, including restructuring the BCV balance sheet through a swap with the pension fund (INPS) of nonmarketable securities for Treasury securities that could be used for monetary operations.

\section{Box 1. The Monetary Policy Framework}

The peg to euro has been Cape Verde's monetary anchor since 1999. ${ }^{1}$ To protect foreign reserves that underpin the exchange rate peg, the BCV uses its policy instruments to influence components of the balance of payments. Since the breakout of the global financial crisis, the $\mathrm{BCV}$ has maintained a tight monetary stance. The first objective of the tight stance has been to support high bank deposit rates relative to euro area rates to discourage repatriation of emigrant deposits, which account for a significant share of bank deposits. The second objective has been to dampen credit growth to the private sector, thereby reducing the demand for imports and shoring up the balance of payments and reserves.

\section{The BCV's main policy tools consist of the minimum reserve requirement (MRR) and} weekly auctions of BCV bills at the policy rate. The MRR is effective in controlling aggregate liquidity in the short run, while the policy rate appears to impact commercial banks' lending and deposit rates at somewhat longer horizons. ${ }^{2}$ The MRR is currently high, at 16 percent, and the current policy rate, at 4.25 percent, represents a large spread to the Euribor. Sterilization losses associated with the high policy rate have been small so far, partly because unremunerated government deposits and MRR support the BCV's balance sheet.

1 Prior to 1999, the Cape Verde's local currency (escudo) was pegged to a basket of currencies (1979-98) and the Portuguese escudo (1975-78).

2 Source: Recent MCM TA Report.

\section{Macroeconomic Policy Mix: Strengthening Coordination}

\section{The macroeconomic policy mix, with expansionary fiscal policy and tight} monetary policy, creates some medium-run risks. The PIP is setting the groundwork for private sector-led growth, but will need to be phased out to achieve this goal. Tight monetary policy is useful to curtail aggregate demand and suppress imports, but may squeeze the supply potential of the economy if access to credit is insufficient. Over the longer run, the sustainability of reserves and the peg will require continued low domestic government debt and success in expanding the narrow export base to generate foreign currency to repay external debt. 
16. The authorities are working to enhance macroeconomic policy coordination. The authorities agree with staff on the usefulness of close coordination for macroeconomic management and to discuss the policy mix and economic outlook (MEFP 914 ).

\section{Financial Sector Strategy: Enhancing the Regulatory Framework}

\section{The BCV commenced publication of a financial stability report, which provides a} comprehensive assessment of risks in the banking sector. The report identifies high credit exposure to real estate and tourism sectors as a key vulnerability. Nonetheless, financial soundness indicators remain favorable (Table 8). Capital adequacy improved, and asset quality remained sound with relatively low NPLs and higher provisioning.

\section{The authorities are making progress to finalize an overarching approach to} safeguard financial stability. The approach will consist of a framework for the whole financial system, rules for the dual banking system, and rules for non-bank financial institutions. The government intends to submit the new banking law to Parliament for approval by December (MEFP $\$ 16$ ). Staff advised the authorities to broaden the range of legally permitted resolution schemes, beyond liquidation (MEFP 917). Staff also encouraged the authorities to finalize a memorandum of understanding (MoU) for the standing financial committee at the BCV.

\section{Staff supports the BCV efforts to enhance competitiveness of the banking sector} and strengthen supervision. The BCV granted a few licenses for onshore banks. Close coordination with home country supervisory agencies is warranted given the large ownership share of Portuguese banks in the domestic banking sector. Nonetheless, the small size of those subsidiaries relative to parent banks reduces the likelihood of funding from domestic to parent banks. The $\mathrm{BCV}$ is in the process of producing a number of regulations to minimize liquidity, exchange rate, and interest risks.

\section{The authorities and staff agree on the importance of moving forward in} developing financial markets. The authorities continue to develop the public debt market by adopting an IT platform (MEFP q15). The National Social Security Institute (INPS) is working with an external consultant to finalize its investment policy guidelines by September 2011.

\section{E. Revitalizing State Owned Enterprises}

21. The government is ready to speed up SOE reforms. The authorities' goal is to put both the electricity company, Electra, and the airline, TACV, on viable restructuring plans (MEFP $911,19,20)$. This would improve their profitability and eventually attract strategic partners from the private sector. Staff encouraged the authorities to continue working with development partners to accelerate reforms in this area. 


\section{F. Improving Labor Market Flexibility}

22. The government is keen to improve performance of labor markets. The authorities are cognizant of the need to enhance human capital and reform labor regulations to promote job creation, particularly among small and medium sized firms. They see economic diversification as creating more suitable job opportunities. However, the government intends to fulfill its election promise of introducing a minimum wage, and is considering how to implement it so as to minimize damage to employment growth and competitiveness.

\section{Box 2. Labor Markets in Cape Verde}

Economic growth in Cape Verde has been capital-intensive, but stronger productivity growth would help spur inclusive growth. Investment-led growth is common for developing economies. As Cape Verde moves up the development ladder, after recently transitioning to middle-income status, further improvements to labor productivity will be needed.

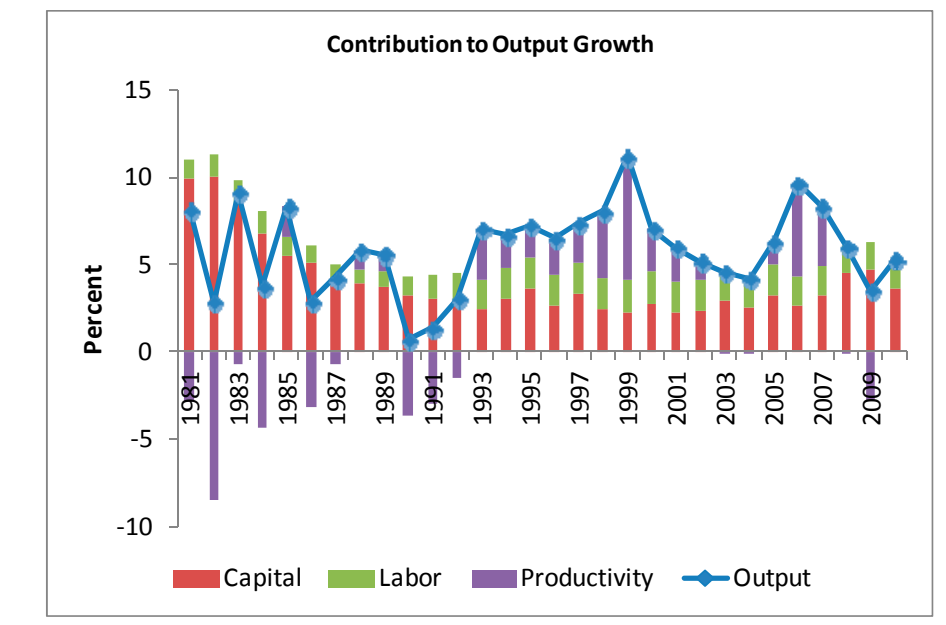

Sources: World Development Indicators (World Bank) and IMF staff estimates.

Labor regulations create rigidities. Rigid contracts and firing regulations continue to constrain competitiveness of businesses, as noted by the World Bank's 'Doing Business.' Such rigidities deter formal job creation and amplify the already large informal sector.

Unemployment is concentrated among youth and women and skill mismatches abound. According to the 2010 Census, the youth unemployment rate (ages 15-24 years) reached 21.3 percent, almost three times higher than older workers (ages 25-64 years). New job opportunities are concentrated in the tourism, construction and commerce sectors, and skills demanded in these sectors are not well matched to skills supplied by the Cape Verdean youth and women. There are also large regional discrepancies in employment prospects and divergent wage and price levels across islands.

The quality of labor supply is uneven. Primary education is widespread and successful. However, the business community notes that secondary school students often lack solid foundations in math and writing, which limit their productivity as workers. Cape Verde has opened several local universities in the past decade, but employers note that well-qualified candidates with tertiary degrees continue to be scarce. Education has also emphasized liberal arts, at the expense of technical training. 


\section{Program Monitoring}

23. The mission reached understandings with the authorities on a proposal to the Board to modify the end-September2011 assessment criterion on net domestic borrowing of the central government. Faster project implementation and stricter-than-expected co-financing requirements associated with some concessional credit lines have increased domestic financing needs in 2011. The new proposed assessment criterion of 2.9 billion escudos represents only a marginal increase that should not undermine fiscal performance.

\section{The structural benchmarks for the remainder of the PSI are shown in MEFP,} Table 2. Nonobservance mainly reflect efforts to improve final outcomes. Following Parliamentary elections in February 2011, the government is preparing a set of related proposals for a comprehensive reform of the tax system, including the income tax bills. The authorities are working with development partners to speed up the process and expect to submit the tax package to Parliament by 2012. The BCV expects to submit the draft banking law to Parliament for approval by December. It is working with an external consultant to further improve the regulation of the off-shore banking sector, which it expects to finalize by July 2011.

25. The coverage for nonconcessional external debt is being broadened to include nonguaranteed external debt from TACV and Electra (TMU, ๆ9), which are the two SOEs that pose fiscal risks.

\section{STAFf ApPraisal}

26. Cape Verde's economic outlook and policy performance remain favorable. The recovery is firmly entrenched, supported by a resilient tourist sector and rapid implementation of the public investment program. The authorities have responded appropriately to the rise in global food and energy shocks by allowing prices to pass through fully into domestic prices, while protecting competitiveness by restraining the second round impact on wages.

\section{While the authorities should complete implementation of the PIP, they should} reduce external debt ratios by avoiding any new external borrowing. With the recovery fully underway, it is important to complete the scaled-up PIP, to provide space for private sector-led growth. A gradual decline of debt ratios after 2012 would also increase resilience against external shocks. Given the already high level of debt, the authorities should avoid incurring any large new projects, especially those that do not relieve constraints on export generation or protect the poor. Any large new projects should be carefully assessed and undergo feasibility studies that include their impact on the debt risk profile.

28. Staff supports efforts to rationalize and simplify the tax system and improve tax administration. Such reforms would help mobilize domestic resources and provide a fair and transparent environment. The government should also carefully monitor revenue outturns and act promptly to keep the fiscal deficit in check. 
29. The tight stance of monetary policy is constraining the growth of private credit to stabilize foreign reserves. The cautious monetary stance is understandable given the uncertain external environment, but staff sees room to relax the stance as reserves accumulate. Staff also commends the BCV's recent efforts to enhance liquidity management and encourages the $\mathrm{BCV}$ to further improve the effectiveness of monetary policy, and develop interbank and government debt markets.

30. Staff welcomes current efforts to coordinate fiscal and monetary policies, including with a view to addressing medium-run risks stemming from current policy mix. Staff encourages the authorities to further enhance coordination to improve macroeconomic management and develop an optimal policy mix for sustainable development.

\section{Staff encourages the authorities to expedite adopting the new banking law and} finalizing the National Social Security Institute's investment policy guidelines. Deepening financial markets and safeguarding the financial system are critical elements for sustainable economic development.

\section{Enhancing labor market flexibility will support the government's transformation} agenda. Rigidities in contracts may hamper the business sector and discourage employment growth, and reforms in these areas would be welcome. The minimum wage could add to labor market rigidities and should be implemented so as to reduce the negative impact on employment and competitiveness. Staff also encourages the authorities to continue accelerating reforms to public enterprises, particularly Electra and TACV.

33. Risks to the program remain manageable. Prospects for economic activity continue to be positive despite the on-going turbulence in Europe. The authorities remain committed to macroeconomic stability and implementation of the program.

34. Staff recommends completion of the first review under the PSI. Staff supports granting a waiver for the two missed end-March 2011 assessment criteria, which reflected temporary and minor factors. Based on the strength of the authorities' policies, staff also supports the proposed modification of the end-September assessment criterion on net domestic borrowing of the central government, the assessment criteria on non-concessional external debt of the central government, and the revisions to the timing of structural benchmarks. 
Figure 1. Cape Verde: Fiscal Performance (Percent of GDP)

The fiscal deficit is peaking in $2011 \ldots$

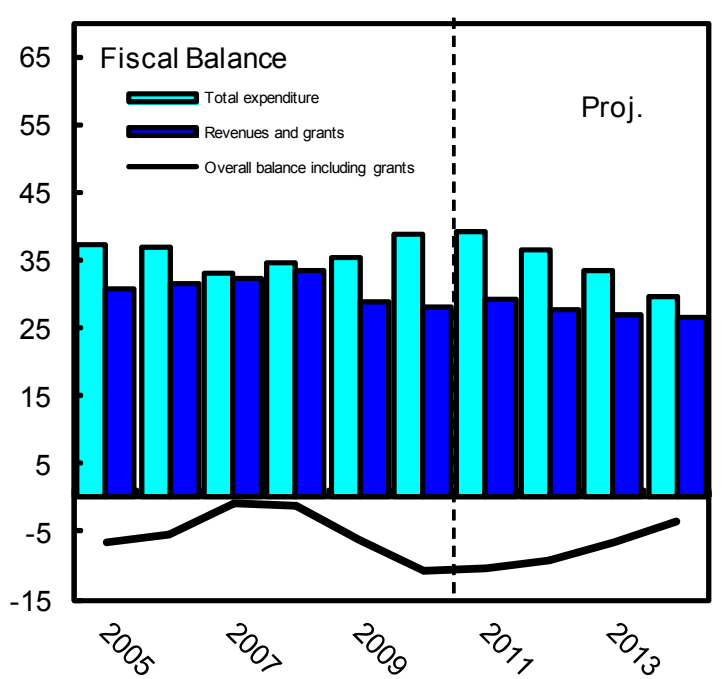

Tax revenues stabilized, broadly covering recurrent expenditures.

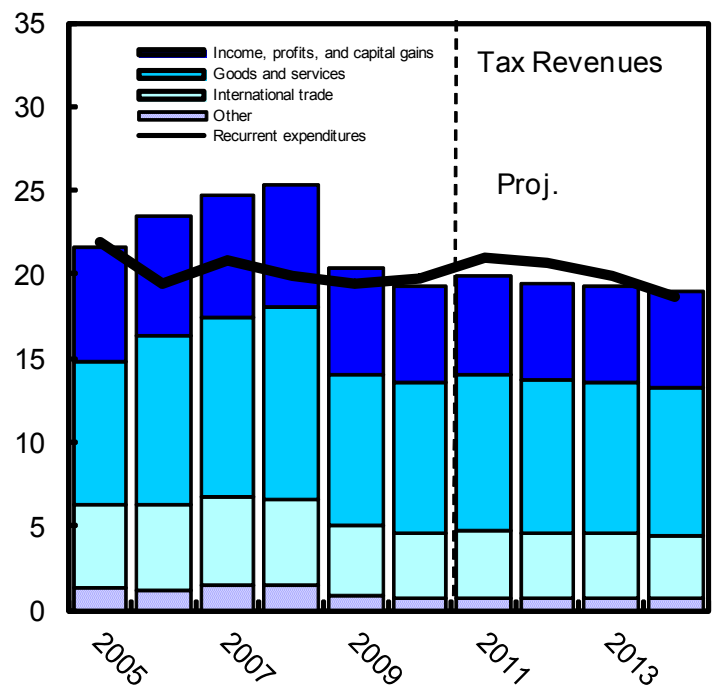

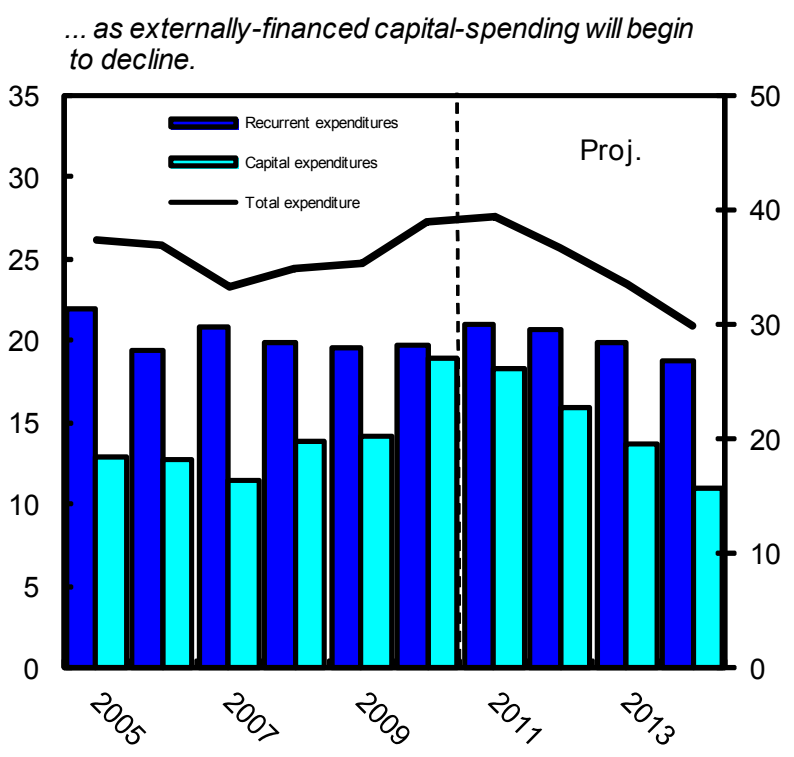

Net domestic debt will continue to be low and support a gradual rise in gross international reserves.

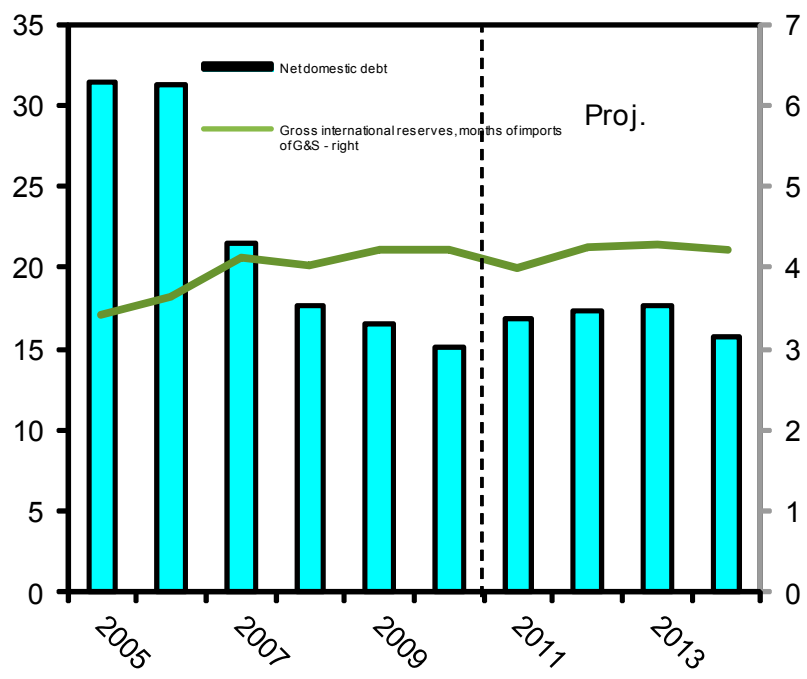

Sources: Cape Verdean auth orities and IMF staff estimates. 
Figure 2. Cape Verde: Monetary Developments

Net foreign assets rebounded since late 2009, but declined slightly in 2011Q1...

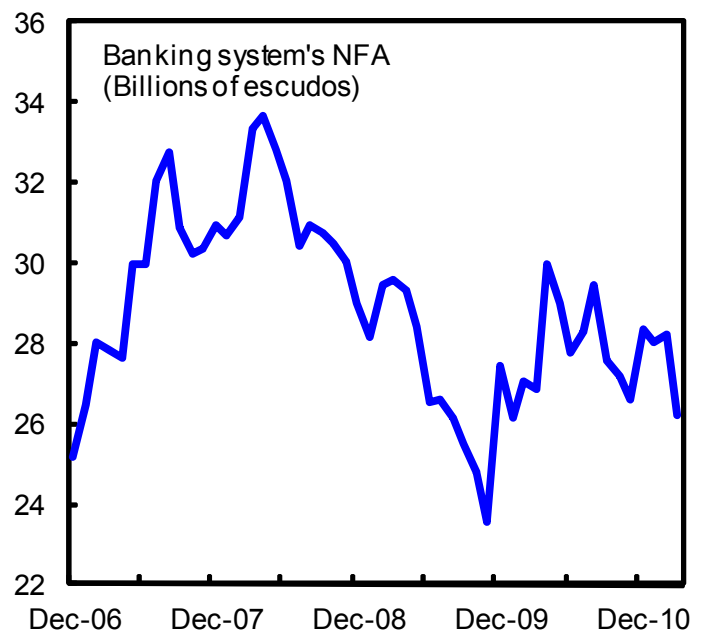

The growth of credit to the private sector has been well below pre-crisis rates...

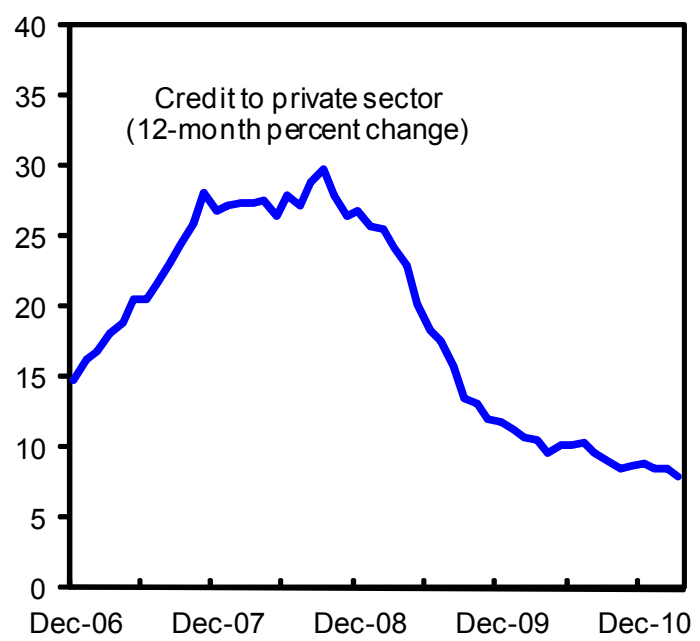

Source: Bank of Cape Verde.
... and the central bank kept its policy rate fixed since January 2010.

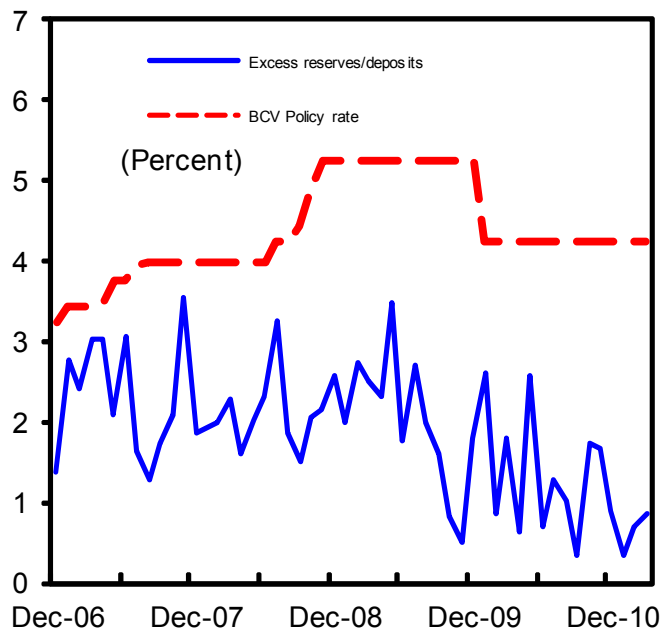

... but the growth rate of broad money edged up slightly.

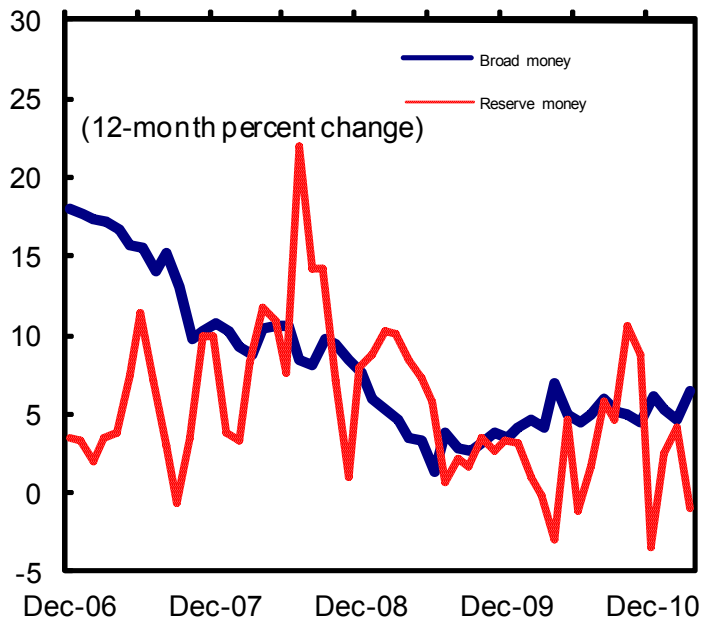


Figure 3. Cape Verde: External Sector Developments

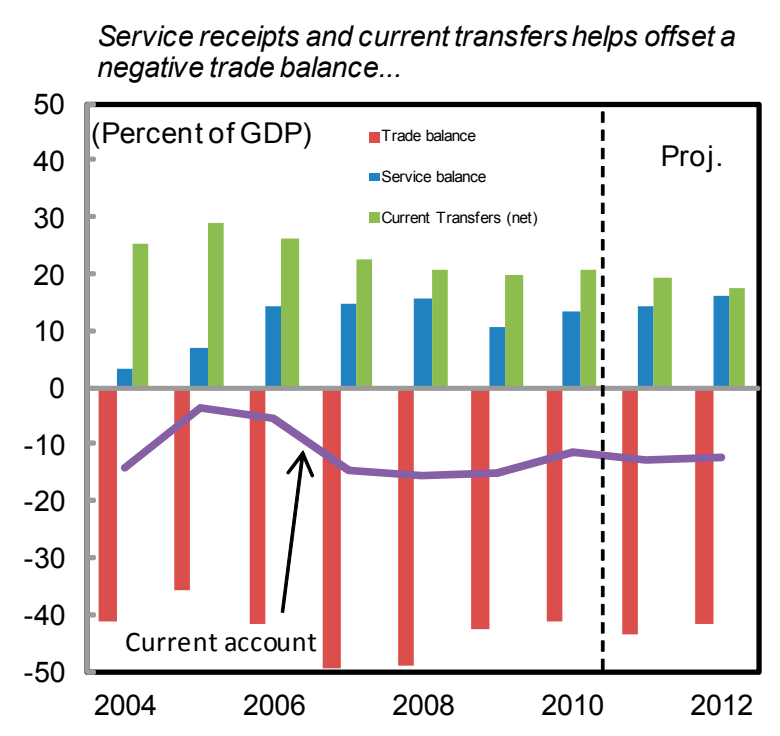

... and the current account deficit was mainly driven by infrastructure-related imports.

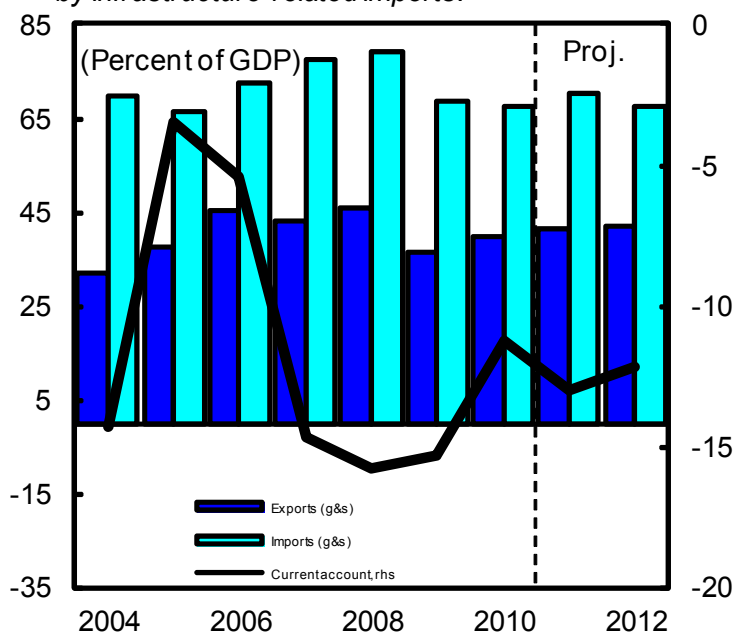

FDI, grants, and concessional loans will finance the current account deficit...
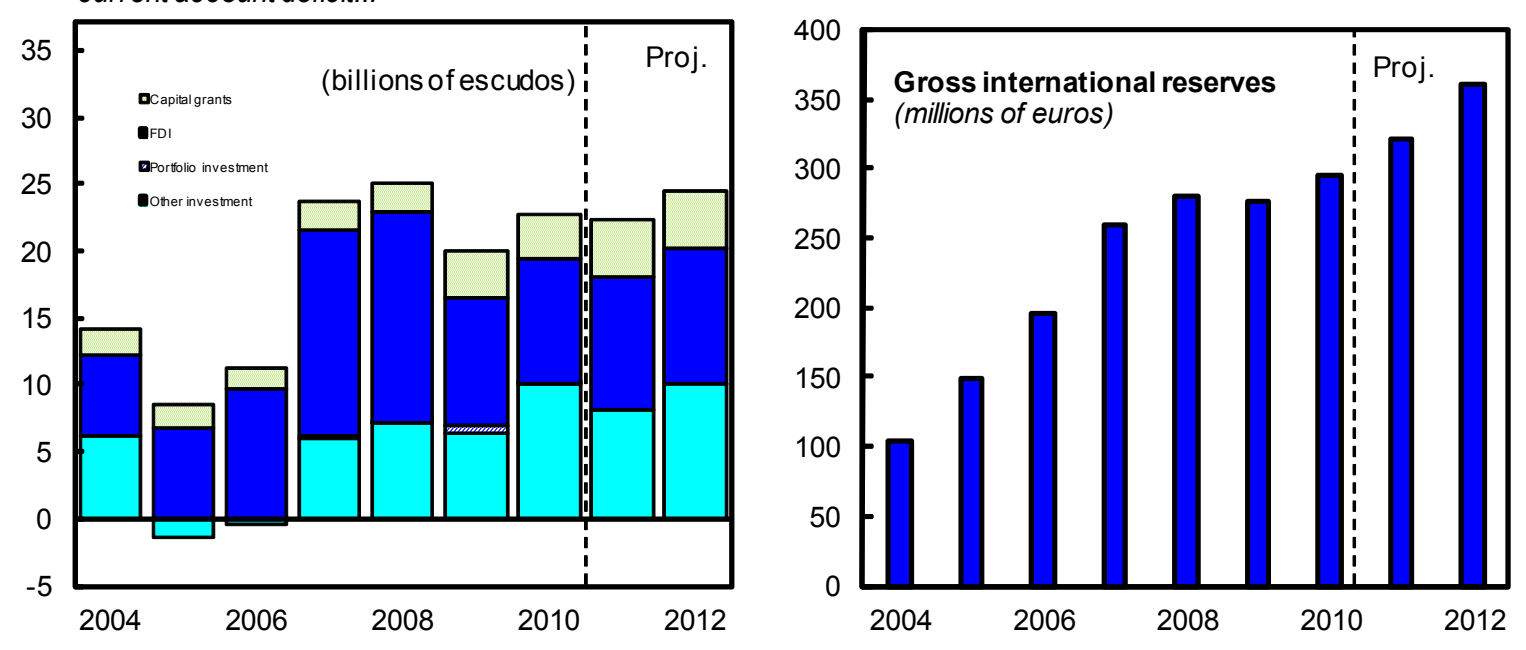

Sources: Cape Verdean auth orities and IMF staff estimates. 
Table 1. Cape Verde Selected Economic Indicators 2009-15

\begin{tabular}{|c|c|c|c|c|c|c|c|}
\hline & 2009 & 2010 & 2011 & 2012 & 2013 & 2014 & 2015 \\
\hline & & & Prog. & \multicolumn{4}{|c|}{ Proj. } \\
\hline & \multicolumn{7}{|c|}{ (Annual Percentage Change) } \\
\hline \multicolumn{8}{|l|}{ National accounts and prices } \\
\hline Real GDP & 3.6 & 5.4 & 5.6 & 6.4 & 6.2 & 6.0 & 5.0 \\
\hline Real GDP per capita & 0.7 & 3.4 & 2.6 & 3.5 & 3.3 & 3.1 & 2.0 \\
\hline GDP deflator & 4.2 & 3.4 & 3.8 & 3.4 & 2.9 & 3.0 & 3.0 \\
\hline Consumer price index (annual average) & 1.0 & 2.1 & 5.2 & 4.8 & 2.8 & 2.1 & 2.0 \\
\hline Consumer price index (end of period) & -0.4 & 3.4 & 6.4 & 3.8 & 2.3 & 2.0 & 2.0 \\
\hline \multicolumn{8}{|l|}{ External sector } \\
\hline Exports of goods and services & -14.3 & 18.8 & 14.1 & 11.5 & 12.8 & 13.0 & 13.1 \\
\hline Of which: tourism & -16.2 & 6.7 & 13.4 & 12.9 & 13.3 & 13.6 & 14.1 \\
\hline Imports of goods and services & -6.3 & 7.1 & 14.7 & 5.7 & 8.4 & 8.2 & 7.6 \\
\hline \multicolumn{8}{|l|}{ Government finance } \\
\hline Total revenue (excluding grants) & -9.4 & 1.3 & 17.0 & 8.2 & 9.0 & 6.1 & 0.8 \\
\hline Total expenditure & 9.3 & 19.9 & 11.0 & 2.5 & -0.2 & -2.9 & -6.9 \\
\hline Noncapital expenditure & 9.1 & 2.9 & 15.4 & 8.5 & 4.8 & 2.8 & 4.6 \\
\hline Capital expenditure & 9.6 & 45.5 & 6.4 & -4.4 & -6.6 & -11.3 & -26.3 \\
\hline \multicolumn{8}{|l|}{ Money and credit } \\
\hline Net foreign assets & -5.4 & 7.6 & 14.9 & 12.2 & 10.2 & 6.4 & 3.3 \\
\hline Net domestic assets & 7.0 & 7.1 & 7.0 & 10.6 & 9.0 & 10.2 & 9.9 \\
\hline net claims on the central government & -6.6 & -26.7 & 39.9 & 21.0 & 15.2 & -5.8 & -8.2 \\
\hline credit to the economy & 11.5 & 8.9 & 5.9 & 8.6 & 7.7 & 9.1 & 9.9 \\
\hline Broad money (M2) & 3.5 & 6.2 & 10.2 & 11.1 & 9.3 & 9.2 & 8.1 \\
\hline Income velocity (GDP/M2) & 1.22 & 1.26 & 1.22 & 1.21 & 1.21 & 1.21 & 1.21 \\
\hline \multirow[t]{2}{*}{ Reserve money (M0) } & 3.4 & -3.4 & 12.0 & 9.1 & 8.0 & 8.0 & 9.3 \\
\hline & \multicolumn{7}{|c|}{ (Percentage of GDP, unless otherwise indicated) } \\
\hline \multicolumn{8}{|l|}{ Saving-investment balance } \\
\hline Gross capital formation & 39.4 & 38.1 & 36.6 & 33.6 & 30.7 & 27.6 & 23.2 \\
\hline Government & 14.1 & 18.9 & 18.4 & 16.0 & 13.6 & 11.1 & 7.5 \\
\hline Gross national savings & 24.1 & 26.8 & 23.7 & 21.4 & 19.7 & 17.8 & 15.5 \\
\hline Government & 9.1 & 8.0 & 7.3 & 5.9 & 6.1 & 6.1 & 5.2 \\
\hline External current account (including official current transfers) & -15.3 & -11.3 & -13.0 & -12.2 & -11.0 & -9.9 & -7.7 \\
\hline External current account (excluding official current transfers) & -20.8 & -17.7 & -18.2 & -16.4 & -14.7 & -13.2 & -10.8 \\
\hline Overall balance of payments & -1.0 & 1.3 & 1.9 & 2.6 & 2.1 & 1.3 & 0.9 \\
\hline \multicolumn{8}{|l|}{ Government finance } \\
\hline Total revenue & 23.6 & 21.9 & 23.4 & 23.0 & 22.9 & 22.3 & 20.8 \\
\hline Total external grants & 5.4 & 6.3 & 5.7 & 4.6 & 4.0 & 4.1 & 3.1 \\
\hline Total expenditure & 35.3 & 38.9 & 39.4 & 36.7 & 33.5 & 29.8 & 25.7 \\
\hline Overall balance (excluding grants) & -11.8 & -17.0 & -16.0 & -13.7 & -10.6 & -7.5 & -4.9 \\
\hline Overall balance (including grants) & -6.3 & -10.7 & -10.3 & -9.1 & -6.6 & -3.4 & -1.8 \\
\hline External financing (net) & 5.0 & 10.6 & 9.9 & 8.4 & 6.6 & 4.1 & 1.9 \\
\hline Domestic financing (net) & 0.8 & -0.7 & 0.4 & 0.7 & 0.0 & -0.7 & -0.2 \\
\hline \multicolumn{8}{|l|}{ Public debt } \\
\hline Total nominal government debt ${ }^{1}$ & 63.0 & 66.4 & 67.2 & 72.4 & 74.9 & 72.8 & 69.3 \\
\hline External government debt ${ }^{2}$ & 46.4 & 51.3 & 50.4 & 55.0 & 57.3 & 57.1 & 55.3 \\
\hline Domestic government debt, net of deposits & 16.5 & 15.1 & 16.8 & 17.4 & 17.6 & 15.7 & 14.0 \\
\hline External debt service (percent of exports of goods and services) & 5.4 & 4.6 & 3.8 & 3.9 & 3.8 & 4.1 & 4.1 \\
\hline \multicolumn{8}{|l|}{ Present Value of External Debt - DSA } \\
\hline PV of debt to GDP (risk threshold: $50 \%$ ) & $\ldots$ & $\ldots$ & 46.7 & 48.9 & 49.6 & 49.0 & 47.3 \\
\hline PV of debt-to-revenue ratio ( risk threshold: $300 \%$ ) & $\ldots$ & $\ldots$ & 199.4 & 212.4 & 216.1 & 219.6 & 227.4 \\
\hline PV of debt-to-exports ratio ( risk threshold: $200 \%$ ) & $\ldots$ & $\ldots$ & 113.1 & 116.9 & 114.9 & 109.7 & 101.3 \\
\hline Gross international reserves (€ millions, end of period) & 275.4 & 294.6 & 320.8 & 359.7 & 393.9 & 417.9 & 436.2 \\
\hline Gross international reserves (months of current imports of goods and services) & 4.2 & 4.2 & 4.0 & 4.2 & 4.3 & 4.2 & 4.1 \\
\hline \multicolumn{8}{|l|}{ Memorandum items: } \\
\hline Nominal GDP (billions of Cape Verde escudos) & 126.0 & 137.2 & 150.3 & 165.5 & 180.8 & 197.4 & 213.5 \\
\hline Net domestic borrowing, net of arrears clearance & 0.2 & 0.1 & 3.0 & 2.1 & 1.7 & -0.4 & -0.5 \\
\hline \multicolumn{8}{|l|}{ Exchange rate (Cape Verde escudos per US\$) } \\
\hline Period average & 79.2 & 83.1 & $\ldots$ & $\ldots$ & $\ldots$ & $\ldots$ & $\ldots$ \\
\hline End period & 75.5 & 83.4 & $\ldots$ & $\ldots$ & $\ldots$ & $\ldots$ & $\ldots$ \\
\hline
\end{tabular}

Sources: Cape Verdean authorities, and IMF staff estimates and projections.

${ }^{1}$ Net of central government deposits; including verified stock of domestic and external arrears.

${ }^{2}$ Excluding claims on the offshore Trust Fund. 
Table 2. Cape Verde: Fiscal Operations of the Central Government, 2009-15 (Millions of Cape Verde escudos, unless otherwise indicated)

\begin{tabular}{|c|c|c|c|c|c|c|c|c|c|}
\hline & & \multirow[t]{2}{*}{2009} & \multirow{2}{*}{$\begin{array}{l}2010 \\
\text { Prel. }\end{array}$} & \multicolumn{2}{|c|}{2011} & \multirow{2}{*}{$\frac{2012}{\text { MTFF }^{1}}$} & \multirow{2}{*}{$\frac{2013}{\text { MTFF }}$} & \multirow{2}{*}{$\frac{2014}{\text { MTFF }}$} & \multirow{2}{*}{$\begin{array}{r}2015 \\
\text { Proj. }\end{array}$} \\
\hline & & & & Q1 & Prog. & & & & \\
\hline Revenue & & 36,540 & 38,723 & 8,670 & 43,760 & 45,702 & 48,645 & 52,145 & 51,024 \\
\hline Domestic revenue & & 29,690 & 30,061 & 7,872 & 35,182 & 38,055 & 41,495 & 44,035 & 44,397 \\
\hline Tax revenue & & 25,676 & 26,391 & 7,046 & 29,915 & 32,162 & 34,920 & 37,329 & 37,099 \\
\hline Income and profit taxes & & 8,059 & 7,865 & 2,282 & 8,906 & 9,576 & 10,396 & 11,114 & 12,162 \\
\hline Consumption taxes & & 11,300 & 12,221 & 3,223 & 13,944 & 14,992 & 16,277 & 17,400 & 19,625 \\
\hline International trade taxes & & 5,219 & 5,388 & 1,272 & 5,927 & 6,373 & 6,919 & 7,396 & 3,663 \\
\hline Other taxes & & 1,098 & 916 & 270 & 1,137 & 1,222 & 1,327 & 1,419 & 1,650 \\
\hline Nontax revenue & r & 4,014 & 3,642 & 826 & 5,242 & 5,868 & 6,550 & 6,681 & 7,297 \\
\hline External grants & & 6,850 & 8,662 & 797 & 8,578 & 7,647 & 7,150 & 8,110 & 6,627 \\
\hline Expenditure & & 44,493 & 53,366 & 11,173 & 59,261 & 60,740 & 60,641 & 58,869 & 54,804 \\
\hline Recurrent expenditure & & 24,602 & 27,157 & 6,590 & 31,479 & 34,348 & 35,990 & 37,008 & 38,699 \\
\hline Primary recurrent expenditure & & 22,705 & 24,984 & 6,068 & 28,987 & 31,867 & 33,364 & 34,228 & 36,327 \\
\hline Wages and salaries & & 14,844 & 16,180 & 4,116 & 18,206 & 19,769 & 20,445 & 21,332 & 23,292 \\
\hline Goods and services & & 2,341 & 2,613 & 405 & 3,425 & 3,362 & 3,450 & 3,657 & 3,955 \\
\hline Transfers and subsidies & & 4,874 & 5,140 & 1,265 & 5,795 & 6,011 & 6,213 & 6,430 & 6,954 \\
\hline Other expenditures & & 646 & 1,051 & 282 & 1,561 & 2,726 & 3,256 & 2,808 & 2,126 \\
\hline Domestic interest payments & & 1,305 & 1,476 & 312 & 1,505 & 1,172 & 1,023 & 1,083 & 1,061 \\
\hline External interest payments & & 592 & 698 & 210 & 988 & 1,308 & 1,603 & 1,697 & 1,312 \\
\hline Capital expenditure & & 17,823 & 25,926 & 3,750 & 27,596 & 26,392 & 24,650 & 21,860 & 16,105 \\
\hline Foreign financed & & 12,346 & 25,256 & 3,451 & 21,441 & 23,665 & 21,471 & 18,491 & 13,555 \\
\hline Domestically financed & & 5,477 & 670 & 299 & 6,155 & 2,726 & 3,180 & 3,370 & 2,550 \\
\hline Overall balance, including grants & & $-7,953$ & $-14,643$ & $-2,503$ & $-15,501$ & $-15,038$ & $-11,996$ & $-6,724$ & $-3,781$ \\
\hline Financing & & 7,953 & 14,643 & 2,503 & 15,501 & 15,038 & 11,996 & 6,724 & 3,781 \\
\hline Foreign, net & & 6,301 & 14,614 & 2,256 & 14,903 & 13,916 & 11,975 & 8,151 & 4,161 \\
\hline Drawings & & 8,248 & 16,594 & 2,654 & 16,818 & 16,018 & 14,321 & 10,381 & 6,928 \\
\hline Amortization & & $-1,947$ & $-1,981$ & -398 & $-1,915$ & $-2,102$ & $-2,346$ & $-2,230$ & $-2,767$ \\
\hline Domestic, net & & 965 & -941 & -179 & 598 & 1,122 & 21 & $-1,426$ & -380 \\
\hline Of which: net domestic borrowing & & 1,900 & 143 & 2,833 & 4,494 & 3,511 & 3,126 & -872 & $-1,000$ \\
\hline Banking system & & 518 & $-1,419$ & 3,236 & 2,247 & 1,755 & 1,563 & -436 & -500 \\
\hline Nonbanks & & 1,382 & 1,562 & -403 & 2,247 & 1,755 & 1,563 & -436 & -500 \\
\hline Of which: accounts payable, net (o/w onlending) & & -829 & -862 & $-2,966$ & $-3,955$ & $-2,489$ & $-2,061$ & -689 & 0 \\
\hline Net errors and omissions & & 687 & 970 & 426 & 0 & 0 & 0 & 0 & 0 \\
\hline Financing gap & & 0 & 0 & 0 & 0 & 0 & 0 & 0 & 0 \\
\hline \multicolumn{10}{|l|}{ Memorandum items: } \\
\hline Overall balance, including grants & & $-6,278$ & $-14,643$ & -299 & $-15,501$ & $-15,038$ & $-11,996$ & $-6,724$ & $-3,781$ \\
\hline External flows ${ }^{2}$ & & 15,098 & 25,256 & 3,053 & 25,395 & 23,665 & 21,471 & 18,491 & 13,555 \\
\hline External debt service (percent of domestic revenue) & & 8.6 & 8.9 & 7.7 & 8.3 & 9.0 & 9.5 & 8.9 & 9.2 \\
\hline Domestic debt ${ }^{3,4}$ & & 20,830 & 20,773 & 23,467 & 25,267 & 28,778 & 31,904 & 31,032 & 29,952 \\
\hline
\end{tabular}

Sources: Ministry of Finance, Bank of Cape Verde, and IMF staff estimates and projections.

${ }^{1}$ Medium-Term Fiscal Framework

${ }^{2}$ External grants + foreign financing.

${ }^{3}$ Including arrears and accounts payable, net of deposits.

${ }^{4}$ On the basis of the residency of the creditor or the currency of denomination of the debt, following the "Debt Limits in Fund-Supported Programs-Proposed New Guidelines". 
Table 3. Cape Verde: Fiscal Operations of the Central Government, 2009-15 (Percent of GDP)

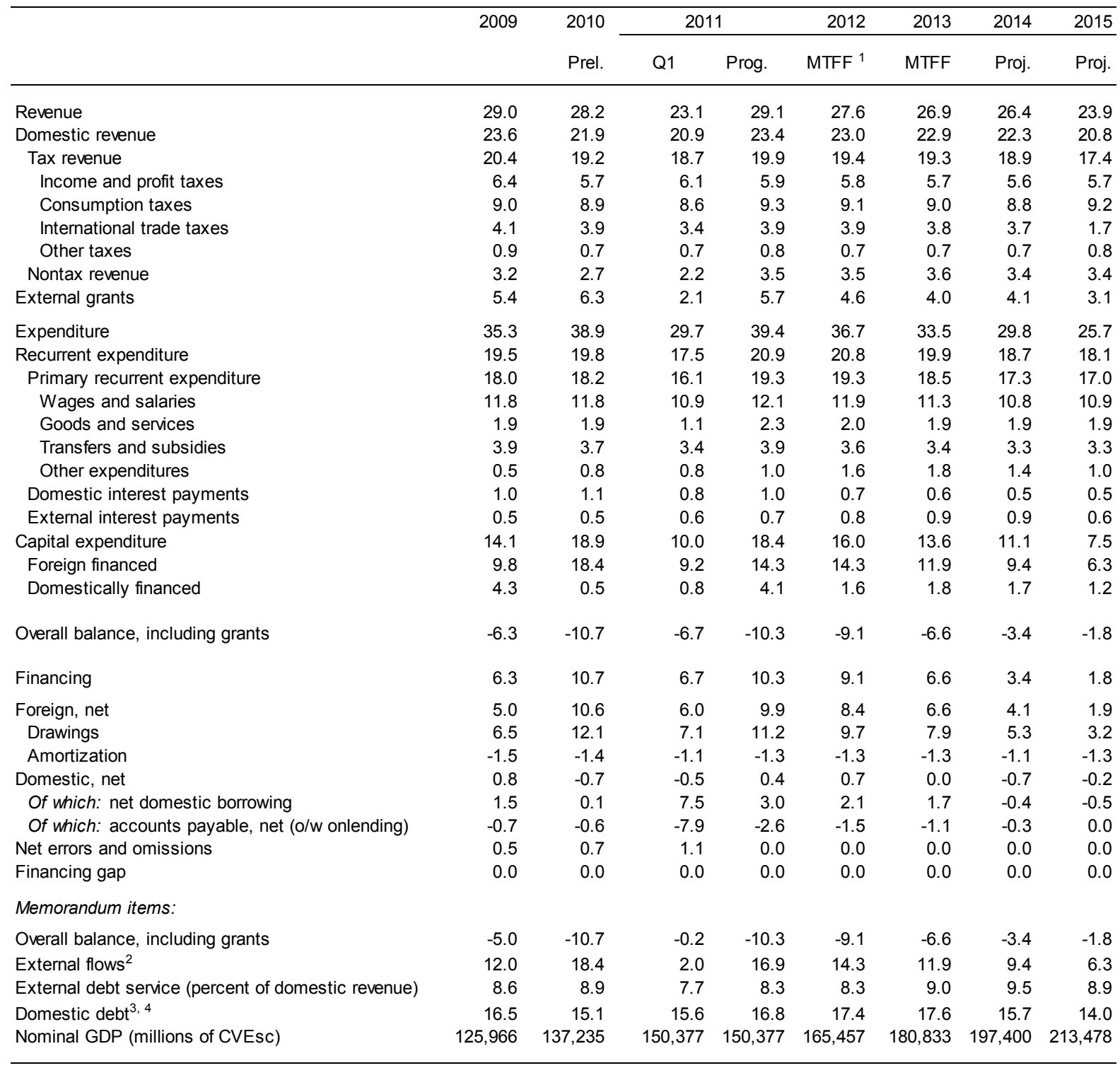

Sources: Ministry of Finance, Bank of Cape Verde, and IMF staff estimates and projections.

${ }^{1}$ Medium-Term Fiscal Framework

${ }^{2}$ External grants + foreign financing.

${ }^{3}$ Including arrears and accounts payable, net of deposits.

${ }^{4}$ On the basis of the residency of the creditor or the currency of denomination of the debt, following the "Debt Limits in Fund-Supported Programs-Proposed New Guidelines". 
Table 4. Cape Verde: Balance of Payments, 2009-15

(Millions of euros, unless otherwise indicated)

\begin{tabular}{|c|c|c|c|c|c|c|c|}
\hline & \multirow[t]{2}{*}{2009} & \multirow[t]{2}{*}{2010} & 2011 & 2012 & 2013 & 2014 & 2015 \\
\hline & & & Prog. & \multicolumn{4}{|c|}{ Proj. } \\
\hline Current account balance (including official transfers) & -175 & -140 & -177 & -183 & -180 & -177 & -149 \\
\hline Trade balance & -487 & -513 & -592 & -627 & -659 & -706 & -764 \\
\hline Exports, f.o.b. & 67 & 102 & 120 & 132 & 149 & 166 & 185 \\
\hline Imports, f.o.b. & -554 & -615 & -712 & -760 & -807 & -873 & -949 \\
\hline Services (net) & 120 & 168 & 194 & 239 & 265 & 314 & 386 \\
\hline Receipt & 348 & 391 & 442 & 495 & 559 & 633 & 719 \\
\hline Of which: tourism & 193 & 206 & 233 & 263 & 298 & 339 & 387 \\
\hline Payment & -228 & -223 & -248 & -256 & -293 & -319 & -333 \\
\hline Income (net) & -31 & -52 & -41 & -53 & -58 & -63 & -64 \\
\hline Credit & 16 & 11 & 15 & 17 & 19 & 21 & 24 \\
\hline Debit & -47 & -63 & -56 & -70 & -77 & -83 & -87 \\
\hline Government interest & -5 & -6 & -4 & -8 & -11 & -13 & -12 \\
\hline Interest by other sectors & -16 & -17 & -23 & -24 & -25 & -27 & -28 \\
\hline Income on direct investment and other income & -27 & -40 & -29 & -37 & -41 & -44 & -47 \\
\hline Current transfers (net) & 224 & 257 & 263 & 259 & 272 & 278 & 293 \\
\hline Government & 63 & 80 & 72 & 63 & 62 & 59 & 60 \\
\hline Others (incl. emigrant remittances) & 161 & 177 & 191 & 196 & 210 & 219 & 233 \\
\hline Capital and financial account (net) & 181 & 204 & 203 & 221 & 214 & 201 & 167 \\
\hline Capital account & 32 & 29 & 40 & 38 & 33 & 63 & 42 \\
\hline \multicolumn{8}{|l|}{ Financial account } \\
\hline Direct investment (net) & 86 & 84 & 90 & 92 & 101 & 101 & 109 \\
\hline Portfolio investment & 4 & 0 & 0 & 0 & 0 & 0 & 0 \\
\hline Other investments, central government & 57 & 133 & 135 & 129 & 114 & 74 & 38 \\
\hline Net official flows & 57 & 133 & 135 & 129 & 114 & 74 & 38 \\
\hline Disbursements & 75 & 150 & 153 & 145 & 130 & 94 & 63 \\
\hline Amortization & -18 & -17 & -17 & -16 & -16 & -20 & -25 \\
\hline Other investments, noncentral government & 2 & -42 & -62 & -38 & -34 & -37 & -22 \\
\hline Commercial banks (net) & 8 & -1 & -4 & 3 & 0 & 0 & 1 \\
\hline Commercial Receipt (net) & 4 & -2 & 0 & 0 & 0 & 0 & 0 \\
\hline Other & -10 & -40 & -58 & -41 & -34 & -37 & -22 \\
\hline Errors and omissions & -18 & -48 & 0 & 0 & 0 & 0 & 0 \\
\hline Overall balance & -12 & 15 & 26 & 39 & 34 & 24 & 18 \\
\hline Financing & 12 & -15 & -26 & -39 & -34 & -24 & -18 \\
\hline Gross international reserves (- accumulation) & 4 & -20 & -26 & -39 & -34 & -24 & -18 \\
\hline Exceptional financing & 8 & 5 & 0 & 0 & 0 & 0 & 0 \\
\hline Financing gap & 0 & 0 & 0 & 0 & 0 & 0 & 0 \\
\hline \multicolumn{8}{|l|}{ Memorandum items: } \\
\hline Current account (including official transfers; percent of GDP) & -15.3 & -11.3 & -13.0 & -12.2 & -11.0 & -9.9 & -7.7 \\
\hline Current account (excluding official transfers; percent of GDP) & -20.8 & -17.7 & -18.2 & -16.4 & -14.7 & -13.2 & -10.8 \\
\hline Overall balance (percent of GDP) & -1.0 & 1.2 & 1.9 & 2.6 & 2.1 & 1.3 & 0.9 \\
\hline Gross international reserves & 275 & 295 & 321 & 360 & 394 & 418 & 436 \\
\hline Months of current year's import of goods and services & 4.2 & 4.2 & 4.0 & 4.2 & 4.3 & 4.2 & 4.1 \\
\hline Months of next year's import of goods and services & 3.9 & 3.7 & 3.8 & 3.9 & 4.0 & 3.9 & 3.7 \\
\hline External public debt & 531 & 639 & 688 & 826 & 939 & 1,022 & 1,070 \\
\hline
\end{tabular}

Sources: Bank of Cape Verde; and IMF staff estimates and projections. 
Table 5. Cape Verde: Monetary Survey, 2009-15

\begin{tabular}{|c|c|c|c|c|c|c|c|}
\hline & 2009 & 2010 & 2011 & 2012 & 2013 & 2014 & 2015 \\
\hline & & & Prog. & \multicolumn{4}{|c|}{ Proj. } \\
\hline & \multicolumn{7}{|c|}{ (Millions of Cape Verde escudos, unless otherw ise specified) } \\
\hline Net foreign assets & 27,414 & 28,379 & 33,892 & 38,032 & 41,902 & 44,585 & 46,075 \\
\hline $\begin{array}{l}\text { Foreign assets } \\
\text { Of which: Gross international reserves } \\
\text { Foreign liabilities }\end{array}$ & $\begin{array}{r}37,614 \\
30,371 \\
-10,200\end{array}$ & $\begin{array}{r}39,762 \\
32,486 \\
-10,277\end{array}$ & $\begin{array}{l}41,855 \\
35,370 \\
-7,963\end{array}$ & $\begin{array}{l}46,770 \\
39,661 \\
-8,737\end{array}$ & $\begin{array}{r}50,637 \\
43,433 \\
-8,734\end{array}$ & $\begin{array}{l}53,379 \\
46,077 \\
-8,794\end{array}$ & $\begin{array}{l}55,493 \\
48,092 \\
-9,418\end{array}$ \\
\hline Net domestic assets & 77,893 & 83,458 & 89,340 & 98,823 & 107,671 & 118,692 & 130,500 \\
\hline Net domestic credit & 97,821 & 103,907 & 111,294 & 120,912 & 130,101 & 139,364 & 150,251 \\
\hline Net claims on general government & 18,599 & 17,636 & 19,937 & 21,693 & 23,256 & 22,820 & 22,177 \\
\hline Claims on the Trust Fund (TCMFs) & 11,038 & 11,584 & 11,584 & 11,584 & 11,584 & 11,584 & 11,584 \\
\hline Net claims on the central government & 7,403 & 5,426 & 7,589 & 9,184 & 10,583 & 9,970 & 9,156 \\
\hline Credit to central government & 15,223 & 15,501 & 17,664 & 19,258 & 20,658 & 20,045 & 19,374 \\
\hline Deposits of central government & $-7,820$ & $-10,075$ & $-10,075$ & $-10,075$ & $-10,075$ & $-10,075$ & $-10,218$ \\
\hline Net claims on local government & 1,052 & 1,544 & 1,691 & 1,861 & 2,034 & 2,221 & 2,402 \\
\hline Net claims on other government agencies (INPS) & -894 & -918 & -927 & -936 & -946 & -955 & -965 \\
\hline Credit to the economy & 79,222 & 86,271 & 91,357 & 99,219 & 106,845 & 116,544 & 128,074 \\
\hline Credit to private sector & 78,749 & 85,761 & 90,847 & 98,709 & 106,335 & 116,034 & 127,564 \\
\hline Claims on nonbank financial institutions & 473 & 510 & 510 & 510 & 510 & 510 & 510 \\
\hline Other items (net) & $-19,928$ & $-20,450$ & $-21,954$ & $-22,089$ & $-22,430$ & $-20,673$ & $-19,751$ \\
\hline Broad money (M2) & 105,307 & 111,838 & 123,232 & 136,855 & 149,573 & 163,276 & 176,575 \\
\hline Narrow money (M1) & 43,014 & 47,022 & 51,803 & 57,530 & 62,876 & 68,637 & 74,227 \\
\hline Currency outside banks & 8,362 & 8,299 & 8,160 & 8,297 & 8,483 & 8,686 & 8,834 \\
\hline Demand deposits & 34,652 & 38,723 & 43,643 & 49,233 & 54,393 & 59,951 & 65,393 \\
\hline Quasi-money & 57,700 & 60,382 & 66,544 & 73,901 & 80,768 & 88,168 & 95,349 \\
\hline \multirow[t]{2}{*}{ Foreign currency deposits } & 4,593 & 4,433 & 4,884 & 5,424 & 5,929 & 6,472 & 6,999 \\
\hline & \multicolumn{7}{|c|}{ (Change in percent of broad money 12 months earlier) } \\
\hline Net foreign assets & -1.5 & 0.9 & 4.9 & 3.4 & 2.8 & 1.8 & 0.9 \\
\hline Net domestic assets & 5.0 & 5.3 & 5.3 & 7.7 & 6.5 & 7.4 & 7.2 \\
\hline Net domestic credit & 7.9 & 5.8 & 6.6 & 7.8 & 6.7 & 6.2 & 6.7 \\
\hline Net claims on the central government & -0.5 & -1.9 & 1.9 & 1.3 & 1.0 & -0.4 & -0.5 \\
\hline Credit to the economy & 8.0 & 6.7 & 4.5 & 6.4 & 5.6 & 6.5 & 7.1 \\
\hline Credit to private sector & 8.2 & 6.7 & 4.5 & 6.4 & 5.6 & 6.5 & 7.1 \\
\hline Other items (net) & -2.9 & -0.5 & -1.3 & -0.1 & -0.2 & 1.2 & 0.6 \\
\hline Broad money (M2) & 3.5 & 6.2 & 10.2 & 11.1 & 9.3 & 9.2 & 8.1 \\
\hline \multicolumn{8}{|l|}{ Memorandum items: } \\
\hline Income velocity of money & 1.22 & 1.26 & 1.22 & 1.21 & 1.21 & 1.21 & 1.21 \\
\hline Emigrant deposits (as percent total deposits) & 37.1 & 37.1 & 36.9 & 36.8 & 36.7 & 36.6 & 36.6 \\
\hline Excess reserves/total deposits (percent) & 1.8 & 0.9 & 0.9 & 0.9 & 0.9 & 0.9 & 1.2 \\
\hline Money multiplier (M2/M0) & 4.0 & 4.0 & 4.2 & 4.3 & 4.4 & 4.4 & 4.4 \\
\hline Credit to the economy (percentage change) & 11.5 & 8.9 & 5.9 & 8.6 & 7.7 & 9.1 & 9.9 \\
\hline
\end{tabular}

Sources: Bank of Cape Verde, and IMF staff estimates and projections. 
Table 6. Cape Verde: Central Bank Survey, 2009-15

\begin{tabular}{|c|c|c|c|c|c|c|c|}
\hline & \multirow[t]{2}{*}{2009} & \multirow[t]{2}{*}{2010} & 2011 & 2012 & 2013 & 2014 & 2015 \\
\hline & & & Prog. & \multicolumn{4}{|c|}{ Proj. } \\
\hline & \multicolumn{7}{|c|}{ (Millions of Cape Verde escudos, unless otherw ise specified) } \\
\hline Net foreign assets & 27,962 & 29,902 & 34,014 & 38,500 & 42,424 & 45,160 & 46,706 \\
\hline Of which: net international reserves & 29,476 & 31,742 & 34,900 & 39,387 & 43,310 & 46,046 & 47,592 \\
\hline Foreign assets & 30,507 & 32,631 & 35,514 & 39,806 & 43,578 & 46,222 & 48,237 \\
\hline Foreign liabilities & $-2,545$ & $-2,584$ & $-1,501$ & $-1,306$ & $-1,154$ & $-1,062$ & $-1,531$ \\
\hline Net domestic assets & $-1,128$ & $-3,986$ & $-4,988$ & $-6,820$ & $-8,218$ & $-8,232$ & $-6,351$ \\
\hline Net domestic credit & 1,334 & $-1,356$ & $-2,590$ & $-4,373$ & $-5,723$ & $-5,687$ & $-3,755$ \\
\hline Trust Fund claims & 4,605 & 4,858 & 4,858 & 4,858 & 4,858 & 4,858 & 4,858 \\
\hline Net claims on central government & $-1,431$ & $-4,158$ & $-4,158$ & $-4,158$ & $-4,158$ & $-4,158$ & $-4,301$ \\
\hline Credit to central government & 3,640 & 2,992 & 2,992 & 2,992 & 2,992 & 2,992 & 2,992 \\
\hline Deposits of central government & $-5,071$ & $-7,150$ & $-7,150$ & $-7,150$ & $-7,150$ & $-7,150$ & $-7,293$ \\
\hline Credit to the economy & 1,099 & 1,308 & 1,314 & 1,321 & 1,327 & 1,334 & 1,341 \\
\hline Credit to private sector & 1,099 & 1,307 & 1,314 & 1,321 & 1,327 & 1,334 & 1,340 \\
\hline Claims on nonbank financial institutions & 0 & 0 & 0 & 0 & 0 & 0 & 0 \\
\hline Net Credit to commercial banks & $-2,939$ & $-3,363$ & $-4,603$ & $-6,393$ & $-7,749$ & $-7,720$ & $-5,652$ \\
\hline Other items (net) & $-2,462$ & $-2,630$ & $-2,398$ & $-2,446$ & $-2,495$ & $-2,545$ & $-2,596$ \\
\hline Reserve money (M0) & 26,834 & 25,916 & 29,025 & 31,681 & 34,206 & 36,928 & 40,355 \\
\hline Currency issued & 10,190 & 10,263 & 10,647 & 11,063 & 11,512 & 12,223 & 12,653 \\
\hline Deposits of commercial banks & 16,984 & 15,726 & 18,763 & 21,034 & 23,143 & 25,416 & 28,133 \\
\hline \multicolumn{8}{|l|}{ Memorandum items: } \\
\hline Gross international reserves (millions of euros) & 275.4 & 294.6 & 320.8 & 359.7 & 393.9 & 417.9 & 436.2 \\
\hline Net international reserves (millions of euros) & 267.3 & 287.9 & 316.5 & 357.2 & 392.8 & 417.6 & 431.6 \\
\hline (months of current imports) & 3.9 & 3.7 & 3.8 & 3.9 & 4.0 & 4.1 & 4.1 \\
\hline Reserve money (12-month change in percent) & 3.4 & -3.4 & 12.0 & 9.1 & 8.0 & 8.0 & 9.3 \\
\hline
\end{tabular}

Sources: Bank of Cape Verde, and IMF staff estimates and projections. 
Table 7. Cape Verde: Deposit Money Bank Survey, 2009-15

\begin{tabular}{|c|c|c|c|c|c|c|c|}
\hline & 2009 & 2010 & 2011 & 2012 & 2013 & 2014 & 2015 \\
\hline & & & Prog. & \multicolumn{4}{|c|}{ Proj. } \\
\hline & \multicolumn{7}{|c|}{ (Millions of Cape Verde escudos, unless otherw ise specified) } \\
\hline Net foreign assets & -548 & -562 & -122 & -468 & -521 & -575 & -631 \\
\hline Foreign assets & 7,107 & 7,132 & 6,341 & 6,964 & 7,059 & 7,156 & 7,256 \\
\hline Foreign liabilities & $-7,655$ & $-7,693$ & $-6,462$ & $-7,432$ & $-7,580$ & $-7,732$ & $-7,887$ \\
\hline Of which: nonresident deposits & $-4,918$ & $-4,942$ & $-4,152$ & $-4,774$ & $-4,870$ & $-4,967$ & $-5,067$ \\
\hline Net domestic assets & 97,490 & 104,096 & 115,193 & 129,026 & 141,611 & 155,166 & 168,372 \\
\hline Net domestic credit & 113,805 & 122,085 & 134,749 & 148,669 & 161,545 & 173,293 & 185,527 \\
\hline Net claims on general government & 15,425 & 16,991 & 19,238 & 20,993 & 22,556 & 22,120 & 21,620 \\
\hline Trust Fund claims & 6,433 & 6,727 & 6,727 & 6,727 & 6,727 & 6,727 & 6,727 \\
\hline Other government deposits (INPS) & -894 & -918 & -927 & -936 & -946 & -955 & -965 \\
\hline Net claims on central government & 8,834 & 9,584 & 11,747 & 13,342 & 14,741 & 14,128 & 13,457 \\
\hline Claims on central government & 11,583 & 12,509 & 14,672 & 16,267 & 17,666 & 17,053 & 16,382 \\
\hline Deposits of central government & $-2,749$ & $-2,925$ & $-2,925$ & $-2,925$ & $-2,925$ & $-2,925$ & $-2,925$ \\
\hline Net claims on local government & 1,052 & 1,544 & 1,691 & 1,861 & 2,034 & 2,221 & 2,402 \\
\hline Credit to the economy & 78,123 & 84,963 & 90,043 & 97,899 & 105,517 & 115,211 & 126,733 \\
\hline Credit to private sector & 77,650 & 84,454 & 89,533 & 97,389 & 105,008 & 114,701 & 126,224 \\
\hline Claims on nonbank financial institutions & 473 & 510 & 510 & 510 & 510 & 510 & 510 \\
\hline Net claims on the Bank of Cape Verde & 20,257 & 21,145 & 25,469 & 29,777 & 33,472 & 35,962 & 37,173 \\
\hline Other items (net) & $-16,315$ & $-17,989$ & $-19,556$ & $-19,642$ & $-19,934$ & $-18,127$ & $-17,155$ \\
\hline Deposit liabilities to nonbank residents & 96,942 & 103,534 & 115,072 & 128,558 & 141,090 & 154,590 & 167,741 \\
\hline Local currency deposits & 92,349 & 99,101 & 110,187 & 123,134 & 135,161 & 148,119 & 160,742 \\
\hline Demand deposits & 34,649 & 38,719 & 43,643 & 49,233 & 54,393 & 59,951 & 65,393 \\
\hline Of which: emigrant deposits & 5,413 & 5,843 & 6,587 & 7,430 & 8,209 & 9,048 & 9,869 \\
\hline Time deposits & 57,700 & 60,382 & 66,544 & 73,901 & 80,768 & 88,168 & 95,349 \\
\hline Of which: emigrant deposits & 29,217 & 31,208 & 34,385 & 38,186 & 41,734 & 45,558 & 49,269 \\
\hline Foreign currency deposits & 4,593 & 4,433 & 4,884 & 5,424 & 5,929 & 6,472 & 6,999 \\
\hline Of which: emigrant deposits & 1,369 & 1,383 & 1,523 & 1,692 & 1,849 & 2,018 & 2,183 \\
\hline \multicolumn{8}{|l|}{ Memorandum items: } \\
\hline Emigrant deposits (ratio to total deposits) & 0.37 & 0.37 & 0.37 & 0.37 & 0.37 & 0.37 & 0.37 \\
\hline \multicolumn{8}{|l|}{ Composition of emigrant deposits } \\
\hline Local currency & 0.96 & 0.96 & 0.96 & 0.96 & 0.96 & 0.96 & 0.96 \\
\hline Demand & 0.15 & 0.16 & 0.16 & 0.16 & 0.16 & 0.16 & 0.16 \\
\hline Time & 0.81 & 0.84 & 0.84 & 0.84 & 0.84 & 0.84 & 0.84 \\
\hline Foreign currency & 0.04 & 0.04 & 0.04 & 0.04 & 0.04 & 0.04 & 0.04 \\
\hline
\end{tabular}

Sources: Bank of Cape Verde, and IMF staff estimates and projections. 
Table 8. Cape Verde: Financial Soundness of the Banking Sector

(End-year; percent unless otherwise indicated)

\begin{tabular}{|c|c|c|c|c|c|c|c|}
\hline Indicator & 2005 & 2006 & 2007 & 2008 & 2009 & 2010 & Mar-11 \\
\hline \multicolumn{8}{|l|}{ Capital adequacy } \\
\hline Regulatory capital to risk-weighted assets & 12.1 & 11.1 & 11.4 & 12.0 & 11.4 & 15.6 & 15.3 \\
\hline Regulatory tier 1 capital to risk-weighted assets & 13.4 & 11.3 & 11.8 & 11.5 & 11.2 & 14.4 & 13.9 \\
\hline \multicolumn{8}{|l|}{ Asset quality ${ }^{1}$} \\
\hline Nonperforming loans to total loans & 6.3 & 3.9 & 13.5 & 10.0 & 3.9 & 4.0 & 4.5 \\
\hline Nonperforming loans net of provisions to capital & -2.5 & -1.7 & 91.1 & 42.2 & -15.2 & -14.6 & -15.2 \\
\hline Provisions to nonperforming loans & 104.9 & 104.1 & 35.0 & 53.1 & 143.5 & 145.2 & 142.2 \\
\hline \multicolumn{8}{|l|}{ Earnings and profitability } \\
\hline Return on assets & 0.5 & 1.1 & 1.6 & 1.0 & 0.7 & 0.7 & 0.2 \\
\hline Return on equity & 9.7 & 19.7 & 30.1 & 15.8 & 9.8 & 9.9 & 2.4 \\
\hline Interest margin to gross income & 62.7 & 67.2 & 71.3 & 68.0 & 74.9 & 77.0 & 76.8 \\
\hline Noninterest expenses to gross income & 61.2 & 54.5 & 46.1 & 57.3 & 67.0 & 67.0 & 66.6 \\
\hline \multicolumn{8}{|l|}{ Liquidity $^{2}$} \\
\hline Liquid assets to total assets & 46.2 & 18.3 & 17.7 & 16.6 & 16.4 & 12.5 & 11.6 \\
\hline Liquid assets to short-term liabilities & 170.2 & 46.1 & 42.9 & 43.8 & 49.2 & 37.7 & 34.5 \\
\hline
\end{tabular}

Source: Bank of Cape Verde.

${ }^{1}$ The NPL ratio for 2007-08 applied stricter loan classification criteria, where a loan was considered nonperforming if it is only 1 day overdue.

${ }^{2}$ Liquid assets include cash in vault and marketable securities. Short-term liabilities include demand deposits.

Table 9. Cape Verde : Proposed Work Program for 2011

Mission Date Purpose Board Review

November 2011

Second PSI review and possible

end-January 2012

negotiation of a new PSI

CInternational Monetary Fund. Not for Redistribution 
Table 10. Cape Verde: Quantitative Assessment Criteria and Indicative Targets for 2010-11 under the PSI ${ }^{1}$

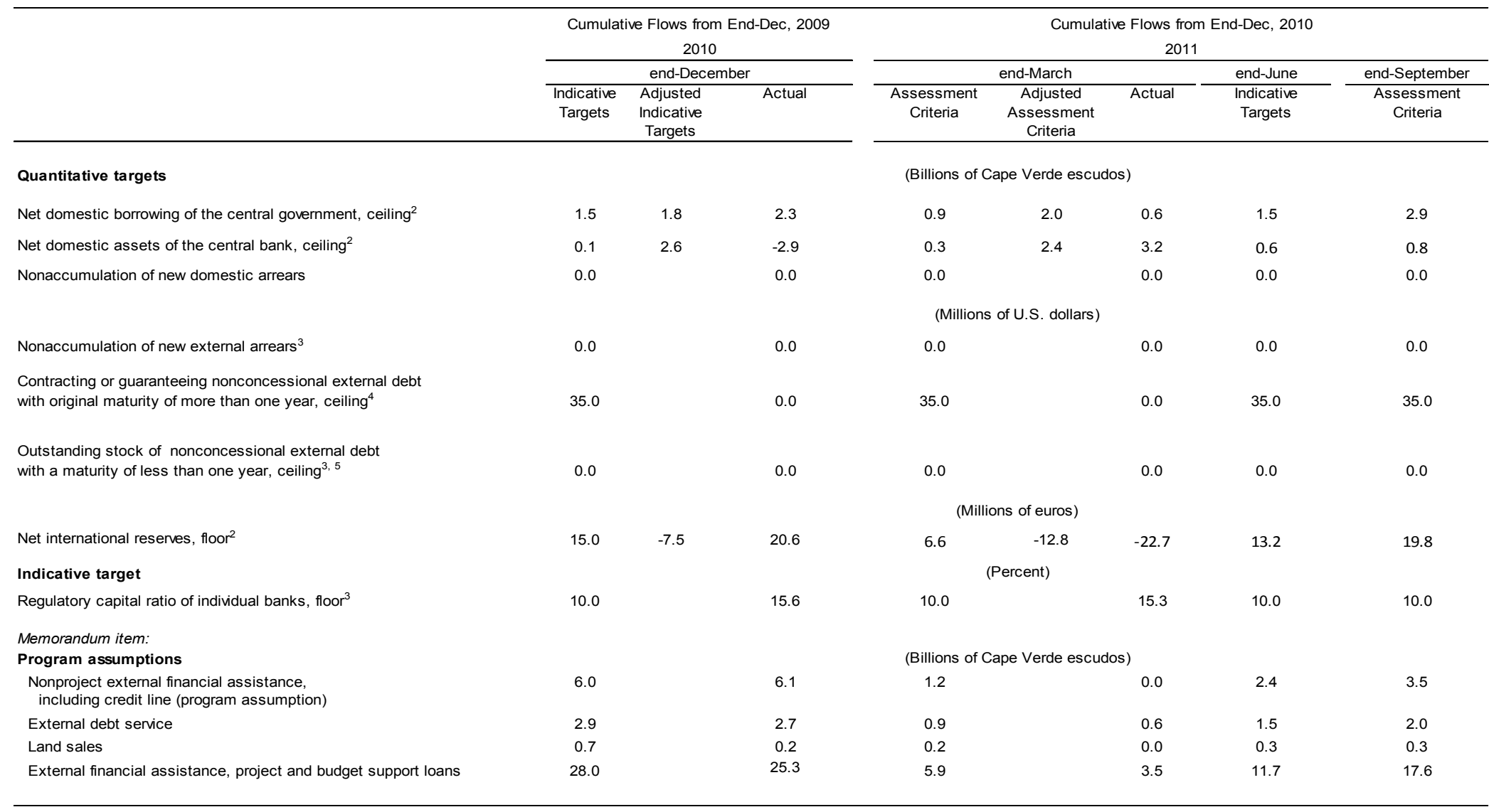

${ }^{1}$ Foreign currency amounts will be converted at current exchange rates.

${ }^{2}$ The ceiling or floor will be adjusted as specified in the TMU.

${ }^{3}$ Continuous.

${ }^{4}$ This assessment criterion applies not only to debt as defined in point No. 9 of the Guidelines on Performance Criteria with Respect to Foreign Debt (Decision No. 12274-(00/85), August 24, 2000, as amended on August 31, 2009), but also commitments contracted or guaranteed for which value has not been received. Excluded from this performance criterion are debt rescheduling and debt reorganization, the Portuguese credit line, and borrowings from the Fund.

${ }^{5}$ The term "debt" has the meaning set forth in point No. 9 of the Guidelines on Performance Criteria with Respect to Foreign Debt (Decision No. 12274-(00/85), August 24, 2000, as amended on August 31, 2009). Excluded from this performance criterion are rescheduling arrangements, the Portuguese credit line, borrowings from the Fund, and import-related financing. 
Table 11. Cape Verde: Structural Benchmarks for 2010-11

\begin{tabular}{|c|c|c|c|}
\hline $\begin{array}{l}\text { Objective/Macro } \\
\text { Criticality }\end{array}$ & Structural Benchmark & Timing & Status \\
\hline $\begin{array}{l}\text { Strengthen fiscal- } \\
\text { monetary policy } \\
\text { coordination }\end{array}$ & $\begin{array}{l}\text { The BCV and Ministry of Finance sign a fiscal-monetary coordination } \\
\text { agreement }\end{array}$ & end-December 2010 & Reset to September 2011 \\
\hline $\begin{array}{l}\text { Strengthen } \\
\text { securities market }\end{array}$ & $\begin{array}{l}\text { Issue fungible treasury bonds with common face and coupon values and } \\
\text { dates of payments }\end{array}$ & end-December 2010 & Reset to June 2011 \\
\hline $\begin{array}{l}\text { Strengthen the } \\
\text { financial sector }\end{array}$ & $\begin{array}{l}\text { The BCV and Ministry of Finance sign a memorandum of understanding to } \\
\text { expand and formalize the standing financial stability committee at the BCV }\end{array}$ & end-March 2011 & Reset to September 2011 \\
\hline $\begin{array}{l}\text { Strengthen debt } \\
\text { management }\end{array}$ & $\begin{array}{l}\text { Create a medium-term strategy report on the debt level, composition, and } \\
\text { desirable trend and present the report to the council of ministers }\end{array}$ & end-June 2011 & Reset to September 2011 \\
\hline Strengthen tax base & $\begin{array}{l}\text { Submit the draft individual and corporate income tax bills to the National } \\
\text { Assembly }\end{array}$ & end-June 2011 & \\
\hline $\begin{array}{l}\text { Promote fiscal } \\
\text { accountability }\end{array}$ & $\begin{array}{l}\text { Present cross debt between SOEs in the contingent liabilities report and } \\
\text { submit at the council of ministers. }\end{array}$ & end-June 2011 & \\
\hline $\begin{array}{l}\text { Strengthen the } \\
\text { financial sector }\end{array}$ & $\begin{array}{l}\text { Submit the new banking law, which unifies the regulatory framework for } \\
\text { onshore and offshore banks, to the National Assembly }\end{array}$ & end-June 2011 & Reset to December 2011 \\
\hline
\end{tabular}

\section{CInternational Monetary Fund. Not for Redistribution}




\section{APPENDIX I. LETTER OF INTENT}

July 5,2011

Christine Lagarde

The Managing Director

International Monetary Fund

$70019^{\text {th }}$ Street N.W.

Washington, D.C. 20431

USA

Dear Ms. Christine Lagarde:

The Cape Verdean government continues to make good progress to implement sound economic and structural policies under the Policy Support Instrument (PSI). In November 22, 2010 the IMF Executive Board approved the request of the government of Cape Verde for a fifteen-month PSI. It is designed to support the government's economic objectives and policy framework for 2010-11. The economic outlook remains favorable as envisaged when the PSI was approved. The government continues to implement key measures to reduce fiscal risks, improve debt management, enhance monetary operations, safeguard the financial sector, and strengthen structural reforms. Details of the economic program being supported by the PSI are set out in the November 2010 Memorandum of Economic and Financial Policies (MEFP) and the attached updated MEFP.

We are committed to implementing the program described in the updated MEFP. It gives particular attention to policy developments and prospects for 2011, and reflects understandings reached with an IMF staff team during the first review mission of the PSI in May 2011. In this update, the government signals its ongoing commitment to pursue macroeconomic and structural policies in support of sustained economic stability, growth, and poverty reduction.

All but one indicate targets were met for end-December 2010, and the quantitative assessment criteria (AC) related to the net domestic borrowing of the central government was met for end-March 2011. The floor on the net international reserves targeted for end-March 2011 was not met because of weaker-than-expected balance of payment performance mainly due to higher import prices and an accelerated repayment of outstanding private external debt. The AC on net domestic assets of the central bank for end-March was not met due to the reduction of the central government deposits at the BCV to implement the public investment program.

We are making good progress toward the three structural benchmarks that have not been observed and request a resetting of these benchmarks. The new IT platform to issue fungible 
government securities is expected to be in place by end-June 2011. The fiscal-monetary coordination agreement has been drafted and an agreement at the technical level has been reached. It will be signed by September 2011. The memorandum of understanding to expand the financial stability committee was discussed, and would be signed by the government by September 2011. The specific quantitative targets and structural measures underlying our adjustment program are summarized in Tables 1 and 2 .

Looking forward to the end-September 2011 assessment criterion, we request a modification of the net domestic borrowing for the central government. Domestic financing needs have increased due to strict co-financing requirements associated with some concessional credit lines. We also request the modification of the definition of nonconcessional external debt of the central government to include the non-guaranteed debt of two state owned enterprises, TACV and Electra.

As committed previously, the government will keep the IMF regularly updated on economic and policy developments and will provide the data needed for adequate monitoring of the program. During the period of the PSI, Cape Verde will consult with the IMF on the adoption of any measures that may be appropriate at the initiative of the government or whenever the Managing Director of the IMF requests such a consultation.

We authorize the IMF to publish this letter, the attached MEFP, and the related staff report.

Sincerely yours,

$/ \mathrm{s} /$

Cristina Duarte

Minister of Finance

Attachments: - Memorandum of Economic and Financial Policies

- Technical Memorandum of Understanding 


\section{Attachment I. Memorandum of Economic and Financial Policies}

\section{INTRODUCTION}

1. This updated Memorandum of Economic and Financial Policies (MEFP) reviews implementation to date of the Cape Verde government's macroeconomic and structural program as set out in the November 2010 Letter of Intent. It describes economic and policy developments since the approval of the PSI and presents the government's main objectives and policy actions for the remainder of the PSI.

2. The government of Cape Verde re-confirms its commitment to pursue sound macroeconomic and structural polices to underpin the country's growth and development. Macroeconomic results and prospects for 2010-11 are broadly in line with expectations at the start of the PSI program. The government is confident that the program will remain on the track.

\section{RECENT ECONOMIC DEVELOPMENTS AND PROGRAM PERFORMANCE}

3. The Cape Verde's economy has been rebounding better than expected. Economic performance has been encouraging, and GDP growth in 2010 is projected to be around 6 percent reflecting stronger-than-expected outcome of the tourism sector and implementation of the public investment program (PIP). Growth should continue to pick up in 2011 benefiting from strong demand in the tourism sector given political turmoil in northern Africa. Economic climate indicators for 2011Q1 shows an increase in economic activities across all sectors. In particular, both the current and perspective activities are mainly favorable in transportation, tourism, and commerce. Employment opportunities are strong in agricultural and construction, especially in the second-home real estate market. In spite of the steady stream of remittances and tourism receipts, the foreign reserves have fallen in the first quarter of 2011. The growth of imports, partly due to price effects, the reductions of the official transfers and foreign direct investment, and the acceleration of the amortization of the outstanding private external debt are the main reasons for the evolution of the foreign reserves in the period.

4. Inflation has edged up in recent months. The 12-month inflation rate increased to 5.2 percent in May 2011, compared to 3.4 percent in December 2010, reflecting the pass-through from the rise in global food and energy prices. Since the beginning of 2011, gasoline and diesel prices increased 13 and 18 percent, respectively. Utility prices for household consumers also increased by 27 percent (electricity) and 15 percent (water). The pass-through to domestic prices could be less than the previous spike in food prices in 2008, given that more agriculture products are now produced in Cape Verde.

\section{Global commodity price shocks and economic turmoil in Europe could pose some} risks to the economic outlook. The recent spike in the global food and oil prices is likely to increase inflationary pressures over the coming months. Bilateral and multilateral financing of credit lines have been steady; however, further austerity measures and economic deterioration in 
the euro area could pose additional risks, mainly to the tourism sector and official financing. Remittances and emigrant deposits have been stable.

6. We have met all but one indicative targets for end-December 2010 and five of seven quantitative assessment criteria (AC) for the end-March 2011, while three structural benchmarks have been delayed with some progress. A fiscal-monetary coordination agreement has been developed and is expect to be signed in by September 2011. Issuance of fungible treasury bonds with common face and coupon values and dates of payments is expected to commence once the IT platform is in place by end-June 2011. Even though phase I of IT platform had already been completed, full implementation has been delayed because of some technical corrections in the system that the project executor in Portugal was asked to complete. A memorandum of understanding to expand and formalize the standing financial stability committee at the BCV has been developed and is expected to be signed by the Minister of Finance and the BCV Governor by September 2011.

\section{Fiscal Developments ANd Policies}

\section{We remain committed to fiscal sustainability and implementation of the medium-} term public investment program. Fiscal developments in 2010 were in line with both objectives. The fiscal deficit reached 10.7 percent of GDP reflecting the higher growth of the public investment program. Recurrent expenditures were swiftly reduced to compensate for weaker-than-expected tax revenues during the year and the overall fiscal balance was lower than projected. For 2011 and 2012, we plan to maintain a steady pace for the public infrastructure investment program in line with our medium-term fiscal framework (MTFF). We have been assured by our partners that concessional financing will be maintained and the partners showed willingness to extend concessional financing until 2015. The government will focus on the implementation of on-going projects, rather than mobilizing resources for new ones.

\section{We are committed to strengthening capacity of the revenue administration and} improving the tax regulatory framework. We acknowledge that there is scope for improving performance of the revenue administration and are dedicated to carry out the necessary diagnostic work to uncover specific issues. We have already identified some areas for improvements including human resources, training, and information systems for revenue management. Additionally, the government is finalizing a long-standing tax reform package for submission to Parliament by 2012 which will revamp the legal framework of the tax system. The individual and corporate income tax bills are part of the necessary reforms and constitute structural benchmarks of the PSI. We will develop a tax exemption bill to rationalize fiscal incentives and level the playing field.

9. The government has taken steps to prepare for any expenditure pressures that materialize from external price shocks. Fiscal space is limited and lower than prior to the 2008/09 global crisis. As a result we are allowing the increase in international food and oil prices to pass on to the consumers, and will limit wage increases. Indeed, there is no provision for wage 
increases for public servants in the 2011 budget. However, we are prepared to use targeted interventions to protect real incomes of the most vulnerable households and safeguard social priority spending. To accomplish this, the government can draw on existing fiscal buffers, reallocate recurrent expenditures, and speed up the use of some existing donor financing linked to social objectives. In doing so, the government expects to address any expenditure needs within the envelope of the MTFF.

10. We continue to improve capacity in debt management and sustainability analysis. A Medium-Term Debt Sustainability (MTDS) exercise with participation from the World Bank and the IMF is underway to improve debt management capacity and provide the basis for preparing the strategy report on public debt. Achievement of the structural benchmark will be delayed until September 2011 due to the timing of the MTDS mission. Additionally, we are receiving technical assistance from the World Bank in fiscal sustainability analysis. The government is committed to enhancing its ability to maintain debt sustainability while promoting the necessary infrastructure investments for the country.

\section{The government continues to take steps to manage fiscal risks from SOEs and is} committed to address the financial difficulties of TACV and ELECTRA. We continue to prepare our contingent liability report currently covering five SOEs. The report provides statistical information and a debt risk assessment for each company, which is then used to inform budgetary provisions for contingent liabilities. This will also contribute to the achievement of End-June structural benchmark on presenting cross debt information among SOEs. For PSI purposes, we will expand coverage of the external non-concessional borrowing limit to include TACV and ELECTRA, which are the SOEs which pose higher fiscal risk.

\section{Monetary and Financial Sector Developments and Policies}

\section{The monetary policy framework continues to be underpinned by the peg, and we} are committed under the PSI to stabilize and protect foreign reserves. The BCV has kept the monetary policy stance tight to maintain emigrant deposits and reserve accumulation, where net international reserves increased by 7.7 percent in 2010. Going forward, we will continue to be vigilant about short-term capital flows and assess their implications for domestic interest rates and credit growth.

\section{The BCV will continue to improve the operational framework for liquidity} management and monetary operations by a number of measures. We intend to: (i) enhance and clarify different roles and instruments of short-term liquidity management and longer-term structural liquidity management; (ii) take measures to create conditions for repo and reverse repo activities; (iii) rationalize the BCV's standing facility to facilitate the interbank market development; (iv) enhance the effectiveness of the monetary transition mechanism; and (v) improve government cash flow forecasting and the BCV's liquidity forecasting to support decisions on liquidity management and monetary operations. 


\section{We are cognizant that good coordination between MoF and BCV are critical for} financial stability, market development, and monetary policy operations. To create enabling institution for market development, a fiscal-monetary coordination agreement between the MoF and BCV has been developed and is expected to be signed in September 2011. Under the agreement, the $\mathrm{BCV}$ and MoF will work together to set up a framework for using treasury securities as an important instrument for monetary operations. We have also developed a memorandum of understanding $(\mathrm{MoU})$ to expand the existing standing financial stability committee at the $\mathrm{BCV}$ to include $\mathrm{MoF}$ to strengthen timely coordination and information exchange between the $\mathrm{MoF}$ and $\mathrm{BCV}$ in crisis response.

\section{We continue our effort to develop an efficient money market and public debt} securities market. We are reviewing and developing necessary market infrastructure to introduce fungible public debt securities, which is expected to be in place by end-June 2011 . We recently started to publish an issuance calendar to strengthen the transparency of the markets. Going forward, we will continue to develop money market and public debt securities markets by: (i) aligning the different tax treatments between treasury securities and BCV securities; (ii) broadening and diversifying the investor base by introducing low-cost modern investment channels such as internet and mobile banking and providing alternative investment opportunities; (iii) enhancing the auction mechanisms by introducing noncompetitive bidding; and (iv) enhancing the market transparency by timely publication of auction results.

16. We are making progress to strengthen the financial sector. The draft legislation of the new banking law harmonizes prudential regulations between onshore and offshore banking activities. The BCV has been working with an external consultant to finalize a dual banking system law by July 2011 to adequately address the supervisory and regulatory framework of the off-shore banking system. We expect the approval of the banking law by the National Assembly by end December 2011. In addition, the BCV has issued its first financial stability report in 2010 and will issue the report on a regular basis in the future. Banks have demonstrated resilience to external shocks. A number of banks increased their equity in 2010. The payment system has made significant progress with substantial increase in the use of ATMs.

\section{Going forward, the $\mathrm{BCV}$ is committed to strengthening the financial stability by} enhancing both micro and macro prudential regulations and the supervisory framework. The BCV will ensure sufficient resources to its supervisory body to effectively carry out both onsite and offsite supervisions. In addition, we will explore and develop comprehensive financial crisis prevention and resolution framework and implementation capacity, which shall incorporate both the latest international experience and our local situation.

\section{STRUCTURAL REFORMS}

\section{The government will embark on a new national agenda for economic}

transformation and social development. Our long term vision is to build a more inclusive, just, and prosperous nation with opportunities for Cape Verdeans, whether in Cape Verde or in the 
Diaspora. To achieve this vision, we will adopt the policy agenda articulated in the government plan for 2011-16 that emphasizes: (i) building a dynamic, competitive, and innovative economy; (ii) facilitating private sector growth, investment, and productivity; (iii) training human resources; (iv) promoting social development; (v) promoting good governance; (vi) modernizing infrastructure; and (vii) affirming our global partnership to increase competitiveness.

\section{We are cognizant that SOE reforms and business diversification are critical for} successful private sector development. SOE reforms slowed down due to the breakout of the global financial crisis. With our economy showing signs of recovery from the global crisis, the National Assembly (Parliament) approved the Law of State Enterprises and Public Managers at the end of 2009, which laid a new legal framework for SOE reforms. As our recovery gains momentum, we are ready to speed up SOE reforms.

\section{We are planning to take some bold actions to put both Electra and TACV on a} viable restructuring plan in order to address their long-standing financial difficulties. The ultimate goal is to allow these companies to improve their profitability so that strategic partners in the private sector are attracted to acquire a stake and allow the companies to play a crucial role in the implementation of the Economic Transformation Agenda. Further improvements in Electra's performance as evidenced by: (i) the adoption of the action plan for the second phase of its institutional restructuring; (ii) the design of a comprehensive, realistic and time-bound approach to the financial restructuring of ELECTRA, including: recapitalization and restructuring of financial short term debt and financing mechanisms for public lighting; (iii) the adoption of a new regulatory tariff-adjustment model compatible with ELECTRA's institutional restructuring; and (iv) the signature of a results-based management contract between ELECTRA and the General Directorate of the. TACV is expected to improve operational, financial and commercial management as evidenced by: (i) the presentation of the audited annual accounts for 2009 and 2010; (ii) the maintenance of zero arrears in the balance for 2010; (iii) full operation of the integrated accounting system for the timely preparation of reports; (iv) signature of a resultbased management contract between the government and TACV; and (v) the adoption of a business plan for 2011-15 by the TACV Board.

\section{Program Monitoring}

21. We are proposing to modify the end-September 2011 assessment criterion on the net domestic borrowing for the central government. The domestic financing needs have increased due to strict co-financing requirements associated with some concessional credit lines. The remaining ACs and structural benchmarks will be maintained. Definitions of terms and specification of reporting requirements are contained in the technical memorandum of understanding (TMU). The second review will be based on the end-September 2011 assessment criteria and is scheduled to be completed by January 31, 2012. 
Table 1. Cape Verde: Quantitative Assessment Criteria and Indicative Targets for 2010-11 under the PSI

\begin{tabular}{|c|c|c|c|c|c|c|c|c|}
\hline & \multirow{2}{*}{\multicolumn{3}{|c|}{$\begin{array}{l}\text { Cumulative Flows from End-Dec, } 2009 \\
2010 \\
\text { end-December }\end{array}$}} & \multicolumn{5}{|c|}{$\begin{array}{l}\text { Cumulative Flows from End-Dec, } 2010 \\
\qquad 2011\end{array}$} \\
\hline & & & & \multicolumn{3}{|c|}{ end-March } & \multirow{2}{*}{$\begin{array}{l}\text { end-June } \\
\text { Indicative } \\
\text { Targets }\end{array}$} & \multirow{2}{*}{$\begin{array}{c}\text { end-September } \\
\text { Assessment } \\
\text { Criteria }\end{array}$} \\
\hline & $\begin{array}{l}\text { Indicative } \\
\text { Targets }\end{array}$ & $\begin{array}{l}\text { Adjusted } \\
\text { Indicative } \\
\text { Targets } \\
\end{array}$ & Actual & $\begin{array}{l}\text { Assessment } \\
\text { Criteria }\end{array}$ & $\begin{array}{c}\text { Adjusted } \\
\text { Assessment } \\
\text { Criteria } \\
\end{array}$ & Actual & & \\
\hline Quantitative targets & \multicolumn{8}{|c|}{ (Billions of Cape Verde escudos) } \\
\hline Net domestic borrowing of the central government, ceiling ${ }^{2}$ & 1.5 & 1.8 & 2.3 & 0.9 & 2.0 & 0.6 & 1.5 & 2.9 \\
\hline Net domestic assets of the central bank, ceiling ${ }^{2}$ & 0.1 & 2.6 & -2.9 & 0.3 & 2.4 & 3.2 & 0.6 & 0.8 \\
\hline Nonaccumulation of new domestic arrears & 0.0 & & 0.0 & 0.0 & & 0.0 & 0.0 & 0.0 \\
\hline & \multicolumn{8}{|c|}{ (Millions of U.S. dollars) } \\
\hline Nonaccumulation of new external arrears ${ }^{3}$ & 0.0 & & 0.0 & 0.0 & & 0.0 & 0.0 & 0.0 \\
\hline $\begin{array}{l}\text { Contracting or guaranteeing nonconcessional external debt } \\
\text { with original maturity of more than one year, ceiling }{ }^{4}\end{array}$ & 35.0 & & 0.0 & 35.0 & & 0.0 & 35.0 & 35.0 \\
\hline \multirow[t]{2}{*}{$\begin{array}{l}\text { Outstanding stock of nonconcessional external debt } \\
\text { with a maturity of less than one year, ceiling }{ }^{3,5}\end{array}$} & 0.0 & & 0.0 & 0.0 & & 0.0 & 0.0 & 0.0 \\
\hline & \multicolumn{8}{|c|}{ (Millions of euros) } \\
\hline Net international reserves, floor ${ }^{2}$ & 15.0 & -7.5 & 20.6 & 6.6 & -12.8 & -22.7 & 13.2 & 19.8 \\
\hline Indicative target & \multicolumn{8}{|c|}{ (Percent) } \\
\hline Regulatory capital ratio of individual banks, floor ${ }^{3}$ & 10.0 & & 15.6 & 10.0 & & 15.3 & 10.0 & 10.0 \\
\hline \multicolumn{9}{|l|}{ Memorandum item: } \\
\hline Program assumptions & \multicolumn{8}{|c|}{ (Billions of Cape Verde escudos) } \\
\hline $\begin{array}{l}\text { Nonproject external financial assistance, } \\
\text { including credit line (program assumption) }\end{array}$ & 6.0 & & 6.1 & 1.2 & & 0.0 & 2.4 & 3.5 \\
\hline External debt service & 2.9 & & 2.7 & 0.9 & & 0.6 & 1.5 & 2.0 \\
\hline Land sales & 0.7 & & 0.2 & 0.2 & & 0.0 & 0.3 & 0.3 \\
\hline External financial assistance, project and budget support loans & 28.0 & & 25.3 & 5.9 & & 3.5 & 11.7 & 17.6 \\
\hline
\end{tabular}

${ }^{1}$ Foreign currency amounts will be converted at current exchange rates.

${ }^{2}$ The ceiling or floor will be adjusted as specified in the TMU.

${ }^{3}$ Continuous.

${ }^{4}$ This assessment criterion applies not only to debt as defined in point No. 9 of the Guidelines on Performance Criteria with Respect to Foreign Debt (Decision No. 12274-(00/85), August 24, 2000, as amended on August 31,2009 ), but also commitments contracted or guaranteed for which value has not been received. Excluded from this performance criterion are debt rescheduling and debt reorganization, the Portuguese credit line, and borrowings from the Fund.

${ }^{5}$ The term "debt" has the meaning set forth in point No. 9 of the Guidelines on Performance Criteria with Respect to Foreign Debt (Decision No. 12274-(00/85), August 24, 2000, as amended on August 31, 2009). Excluded from this performance criterion are rescheduling arrangements, the Portuguese credit line, borrowings from the Fund, and import-related financing. 
Table 2. Cape Verde: Structural Benchmarks for 2010-11

\begin{tabular}{|c|c|c|c|}
\hline $\begin{array}{l}\text { Objective/Macro } \\
\text { Criticality }\end{array}$ & Structural Benchmark & Timing & Status \\
\hline $\begin{array}{l}\text { Strengthen fiscal- } \\
\text { monetary policy } \\
\text { coordination }\end{array}$ & $\begin{array}{l}\text { The BCV and Ministry of Finance sign a fiscal-monetary coordination } \\
\text { agreement }\end{array}$ & end-December 2010 & Reset to September 2011 \\
\hline $\begin{array}{l}\text { Strengthen } \\
\text { securities market }\end{array}$ & $\begin{array}{l}\text { Issue fungible treasury bonds with common face and coupon values and } \\
\text { dates of payments }\end{array}$ & end-December 2010 & Reset to June 2011 \\
\hline $\begin{array}{l}\text { Strengthen the } \\
\text { financial sector }\end{array}$ & $\begin{array}{l}\text { The BCV and Ministry of Finance sign a memorandum of understanding to } \\
\text { expand and formalize the standing financial stability committee at the BCV }\end{array}$ & end-March 2011 & Reset to September 2011 \\
\hline $\begin{array}{l}\text { Strengthen debt } \\
\text { management }\end{array}$ & $\begin{array}{l}\text { Create a medium-term strategy report on the debt level, composition, and } \\
\text { desirable trend and present the report to the council of ministers }\end{array}$ & end-June 2011 & Reset to September 2011 \\
\hline Strengthen tax base & $\begin{array}{l}\text { Submit the draft individual and corporate income tax bills to the National } \\
\text { Assembly }\end{array}$ & end-June 2011 & \\
\hline $\begin{array}{l}\text { Promote fiscal } \\
\text { accountability }\end{array}$ & $\begin{array}{l}\text { Present cross debt between SOEs in the contingent liabilities report and } \\
\text { submit at the council of ministers. }\end{array}$ & end-June 2011 & \\
\hline $\begin{array}{l}\text { Strengthen the } \\
\text { financial sector }\end{array}$ & $\begin{array}{l}\text { Submit the new banking law, which unifies the regulatory framework for } \\
\text { onshore and offshore banks, to the National Assembly }\end{array}$ & end-June 2011 & Reset to December 2011 \\
\hline
\end{tabular}

\section{CInternational Monetary Fund. Not for Redistribution}




\section{Attachment II. Technical Memorandum OF Understanding}

1. This memorandum sets out the understandings between the Cape Verdean authorities and the IMF staff regarding the definition of assessment criteria and indicative targets and reporting requirements for the second review under the Policy Support Instrument.

\section{Quantitative Assessment Criteria ANd Indicative Targets}

\section{A. Net Domestic Borrowing Excluding for Clearance of Arrears and Net Late Payments}

2. The central government includes all units of budgetary central government. It does not include local government (municipalities) and public corporations.

3. Net domestic borrowing excluding for clearance of arrears and net late payments is defined as the cumulative change since the start of the calendar year of the net credit to the central government from the banking and nonbanking sectors less (1) the cumulative clearance during the calendar year of the stock of arrears as of the end of the previous year and (2) the cumulative payments during the first three months of the calendar year of expenses authorized by the previous year's budget, plus the expenses accrued during the current year that will be paid during the first three months of the next calendar year as provisioned for in the budget law (late payments or atrasados).

- $\quad$ The ceiling will be adjusted upward by

$>$ the cumulative upward deviations in external debt service, by

$>$ the cumulative downward deviations in nonproject external financial assistance and land sales relative to program assumptions, and by the costs of restructuring state-owned enterprises up to a limit of CVE 1.5 billion.

- $\quad$ The ceiling will be adjusted downward by

$>$ the cumulative downward deviation in external debt service and by

$>$ the cumulative upward deviation in land sales relative to program assumptions.

4. Net credit to the central government from the banking and nonbanking system is defined as the overall position of the main central government institutions vis-à-vis the banking and nonbanking system - that is, the stock of all outstanding claims on the central government (loans, advances), and all other government debt instruments, such as long-term government securities, held by the $\mathrm{BCV}$, commercial banks, and nonbank institutions, less all deposits held by the central government with the BCV and with commercial banks. The INPS is not included in central government accounts. Net credit to the central government excludes claims on the Trust Fund (TCMFs).

5. Reporting requirements. Data on the implementation of the budget compiled by the Ministry of Finance and Public Administration will be provided on a quarterly basis, to be submitted no later 
than five weeks after the end of each quarter, including (i) government domestic revenue by category; (ii) external budget support grants; (iii) government expenditure, including primary current expenditure, domestic and external interest payments, and capital expenditure, including domestically and budget support financed capital expenditure and estimates of externally project financed capital expenditure; (iv) the gross payment and gross accumulation of domestic accounts payable (atrasados); (v) the gross payment and gross accumulation of domestic payments arrears; (vi) external loan receipts and principal payments; (vii) external arrears payments and accumulation; (viii) bank and nonbank financing; (ix) privatization and land sale receipts; and (x) any other revenue, expenditure, or financing not included above.

6. For the purposes of this memorandum, privatization and land proceeds will be understood to mean all monies received by the government from the sale or concessioning of a public company, organization, or facility to a private company or companies, organization(s), or individual(s), as well as any proceeds generated from the sale of government land and the liquidation of a public company, less restructuring costs.

\section{B. Net Domestic Assets of the Central Bank}

7. The ceiling on the cumulative change, from the beginning of calendar-year 2011, in net domestic assets of the BCV constitutes an assessment criterion. Net domestic assets (NDA) of the $\mathrm{BCV}$ are defined as reserve money minus net foreign assets of the $\mathrm{BCV}$, evaluated at the current end-of-period exchange rates.

- $\quad$ The program ceilings for NDA will be adjusted upward by

$>\quad$ the cumulative upward deviations in external debt service, by

the cumulative downward deviations in external financial assistance, and project and budget loans relative to program assumptions, and by the costs of restructuring state-owned enterprises up to a limit of CVE 1.5 billion.

- $\quad$ The ceiling will be adjusted downward by the cumulative downward deviation in external debt service relative to program assumptions.

For purposes of calculating the adjusters, these flows will be valued at current exchange rates. Reserve money comprises bank reserves and deposits of the monetary institutions and private sector with the central bank, as well as cash in circulation.

8. Reporting requirements. The preliminary monthly balance sheets of the BCV and the consolidated commercial banks will be transmitted on a monthly basis, with a maximum delay of five weeks. The definitive version of the monthly balance sheet of the BCV will be provided as soon as available. 


\section{Ceiling on Nonconcessional External Debt Contracted or Guaranteed by the Central Government}

9. External debt (long-term, medium-term, and short-term) is defined as debt to nonresidents contracted or guaranteed by the central government, the Bank of Cape Verde, and the external debt of the official sector entities and state owned enterprises (SOEs) guaranteed by the central government. External debt also includes the private external debt to nonresidents for which official guarantees have been extended. For the purposes of the relevant assessment criteria, the definition of debt is set out in Executive Board Decision No.6230-(79/140), Point 9, as revised on August 31, 2009 (Decision No. 14416-(09/91)). External debt is defined as debt contracted with nonresidents. The ceiling on nonconcessional external debt applies to external debt contracted or guaranteed by the central government, the Bank of Cape Verde, TACV and ELECTRA.

10. The program's assessment criteria on nonconcessional external debt include ceilings on medium- and long-term, as well as on short-term, nonconcessional external debt. The ceiling on contracting or guaranteeing nonconcessional external debt with original maturity of less than one year by the central government is assessed on a continuous basis. Nonconcessional external debt is defined as debt contracted or guaranteed by the central government, the Bank of Cape Verde, TACV and ELECTRA with a grant element of less than 35 percent, calculated using currencyspecific commercial interest reference rates (CIRRs) published by the Development Assistance Committee of the Organization for Economic Cooperation and Development (OECD). Debt rescheduling and debt reorganization are excluded from the limits on nonconcessional external debt. The assessment criteria on new nonconcessional external debt contracted or guaranteed by the central government, the Bank of Cape Verde, TACV and ELECTRA (excluding borrowing from the Fund) are specified in Table 1 of the Letter of Intent. Nonconcessional external debt with maturity of more than one year excludes normal short-term (less than one year) import-related financing. The Portuguese government's precautionary credit line (the "Portuguese credit line") in support of the exchange rate peg is also excluded from the definition of nonconcessional external debt.

11. Reporting requirements. The government of Cape Verde will consult with Fund staff before assuming any liabilities in circumstances where they are uncertain whether the instrument in question falls under the assessment criteria. Details of all new external debt (including government guarantees), indicating terms of debt and creditors, will be provided on a quarterly basis within five weeks of the end of each quarter.

\section{Net International Reserves of the Central Bank}

12. The floor on the cumulative change, from the beginning of calendar-year 2011, in net international reserves (NIR) of the BCV constitutes an assessment criterion under the program. The NIR of the $\mathrm{BCV}$ are defined as gross international reserves of the $\mathrm{BCV}$ net of its external reserve liabilities, calculated at the current exchange rates. Gross reserves of the BCV are those that are readily available (i.e., liquid and marketable and free of any pledges or encumbrances), controlled by the $\mathrm{BCV}$ and held for the purposes of meeting balance of payments needs and intervening in 
foreign exchange markets. They include gold, holdings of SDRs, the reserve position at the IMF, holdings of foreign exchange and traveler's checks, demand and short-term deposits at foreign banks abroad, fixed-term deposits abroad that can be liquidated without penalty, and any holdings of investment-grade securities. External liabilities of the BCV comprise liabilities to nonresidents contracted by the $\mathrm{BCV}$ with an original maturity of less than a year, any net off-balance-sheet position of the BCV (futures, forwards, swaps, or options) with either residents and nonresidents, any arrears on principal and interest to external creditors and suppliers, and purchases from the IMF.

- $\quad$ The program floors for the NIR will be adjusted downward by

$>$ the cumulative upward deviations in external debt service, by

$>\quad$ the cumulative downward deviations in external financial assistance, and project and budget loans relative to program assumptions, and by the costs of restructuring state owned enterprises up to a limit of $€ 13.6$ million. For purposes of calculating the adjusters, these flows will be valued at current exchange rates.

- The floor will be adjusted upward by the cumulative downward deviation in external debt service relative to program assumptions. Additionally, in the case Cape Verde participates in any SDR allocation(s) between June 30, 2009 and the test date, this floor will be adjusted upwards by the equivalent of the amount of the cumulative additional SDR allocation(s) up to the test date measured at program exchange rates.

13. Reporting requirements. A table on the NIR prepared by the BCV will be transmitted on weekly basis, with a maximum delay of two weeks.

\section{E. Nonaccumulation of New Domestic Payments Arrears}

14. As part of the program, the central government will not accumulate any new domestic payments arrears. This will be monitored through the monthly execution of the cash-flow plan and the corresponding release of budget appropriations. For programming purposes, a domestic payment obligation to suppliers is deemed to be in arrears if it has not been paid within the normal grace period of 60 days ( 30 days for government salaries and debt service) or such other period either specified by the budget law or contractually agreed with the supplier after the verified delivery of the concerned goods and services, unless the amount or the timing of the payment is subject to good faith negotiations between the government and the creditor.

15. Reporting requirements. The Ministry of Finance and Public Administration, through the D.G.T., will submit on a quarterly basis a detailed table of the stock of domestic payments arrears, including the accumulation, payment, rescheduling and write-off of domestic payments arrears during the quarter. The data are to be provided within four weeks after the end of the quarter. 


\section{F. Nonaccumulation of External Payments Arrears}

16. As part of the program, the central government will not accumulate any new external payments arrears on a continuous basis. This will be monitored through the monthly execution of the cash-flow plan and the corresponding release of budget appropriations.

17. External arrears are defined as total external debt-service obligations of the central government that have not been paid by the time they are due, except where agreements between the central government and creditors explicitly provide for a grace period after such obligations falling due. External arrears exclude arrears on external debt, pending the conclusion of debt-rescheduling agreements.

18. Reporting requirements. Data on (i) debt-service payments; and (ii) external arrears accumulation and payments will be transmitted on a quarterly basis by the Ministry of Finance and Public Administration, within five weeks of the end of each quarter. In addition, the central government will inform the Fund staff immediately of any accumulation of external arrears.

\section{G. Regulatory Capital Ratio}

19. The floor is defined as the minimum ratio of regulatory capital to risk-weighted assets following the IMF Financial Soundness Indicators Compilation Guide. This will be monitored through the monthly regulatory capital to risk-weighted assets for each individual bank in the financial soundness indicators of the banking sector. The regulatory capital ratio of individual banks will be provided to the IMF on a monthly basis within six weeks after the end of each month.

\section{OTHER DATA REQUIREMENTS}

20. Data on exports and imports, including volume and prices and compiled by the Director of Customs and the BCV, will be transmitted on a quarterly basis within five weeks after the end of each quarter. A preliminary quarterly balance of payments, compiled by the BCV, will be forwarded within five weeks after the end of each quarter.

21. The Statement of Other Economic Flows as defined in the IMF Manual GFSM2001 relative to holding gains/losses of the previous year with Enapor, Electra, ASA, TACV, and IFH will be transmitted on an annual basis within three months after the end of the following year (15 months after the closing date).

22. The consolidated balance sheet of Electra, Enapor, ASA, TACV and IFH relative to the previous year will be transmitted on an annual basis within three months after the end of the following year (15 months after the closing date).

23. The table of Financial Soundness Indicators will be transmitted to the IMF on a monthly basis within six weeks after the end of each month. The nomination of individual banks is optional. 
Annex I. Cape Verde-Relations with the Fund (as of May 31, 2011)

I. Membership Status: Joined: November 20, 1978;

II. General Resources Account:

Quota

Fund holdings of currency (Exchange Rate)

Reserve Tranche Position

III. SDR Department:

Net cumulative allocation

Holdings

IV. Outstanding Purchases and Loans:

ECF Arrangements
SDR Million

$\underline{\text { Article VIII }}$

\%Quota

100.00

9.59

99.93

0.02

0.17

SDR Million

\%Allocation

9.17

100.00

6.24

68.02

SDR Million

5.07

\%Quota

52.81

V. Latest Financial Arrangements:

Date of Expiration

$\frac{\text { Type }}{\mathrm{ECF}^{\mathbf{1 /}}}$

Stand-By

${ }^{1 /}$ Formerly PRGF. Arrangement Apr 10, 2002 Date Jul 31, 2005 Feb 20, 1998 Mar 15, 2000 Amount Approved Amount Drawn (SDR Million) (SDR Million) 8.64

\section{Projected Payments to Fund ${ }^{2 /}$}

(SDR Million; based on existing use of resources and present holdings of SDRs):

\begin{tabular}{lccccc} 
& \multicolumn{5}{c}{ Forthcoming } \\
\cline { 2 - 6 } & $\underline{2011}$ & $\underline{2012}$ & $\underline{2013}$ & $\underline{2014}$ & $\underline{2015}$ \\
Principal & 0.01 & 0.02 & 0.02 & 0.02 & 0.02 \\
Charges/Interest & $\underline{1.24}$ & $\underline{1.63}$ & $\underline{1.26}$ & $\underline{0.76}$ & $\underline{0.26}$ \\
Total & When a member has overdue financial obligations outstanding for more than three & months, the amount of \\
such arrears will be shown in this section.
\end{tabular}

VII. Implementation of HIPC Initiative: Not Applicable

VIII. Implementation of Multilateral Debt Relief Initiative (MDRI): Not Applicable

\section{Safeguards Assessments}

Safeguards assessments of the Bank of Cape Verde (BCV) were completed in 2002 and 2008. The 2008 assessment was conducted at the request of the authorities. The 2008 assessment found that the BCV had addressed some of the measures recommended in 2002, but also made new recommendations to further strengthen its control and governance framework and transparency practices. The BCV has since improved the timing of publication of the audited results and the 2010 annual financial statements are available on its website. 


\section{Exchange Arrangements}

The de facto and de jure exchange rate arrangement of Cape Verde is a conventional fixed peg. The escudo has been pegged to the euro at a rate of CVE 110.265 per euro since January 4, 1999. Cape Verde accepted the obligations of Article VIII of the Articles of Agreement effective July 1, 2004. It maintains an exchange system that is free of restrictions on the making of payments and transfers for current international transactions.

\section{Previous Article IV Consultation and PSI Reviews}

The Executive Board concluded the Article IV consultation and approved the request for a PSI on July 31, 2006 (Country Report No. 06/334); the first PSI review on January 19, 2007 (Country Report No. 07/44); the second PSI review on May 7, 2007 (Country Report No. 07/223); the third PSI review on December 21, 2007 (Country Report 08/37); the 2008 Article IV consultation and the fourth PSI review on June 30, 2008 (Country Report 08/248); the fifth PSI review on December 22, 2008 (Country Report 09/14); the sixth PSI review on June 19, 2009 (Country Report 09/198); the seventh PSI review on December 18, 2009 (Country Report 09/328); and the eight PSI review on July 19, 2010 (Country Report 10/218). The Executive Board concluded the 2010 Article IV consultation and approved a 15-month PSI arrangement in November 2010 (Country Report 10/349).

\section{Technical Assistance}

Since 1985, Cape Verde has received substantial technical assistance. Technical assistance activities since 2004 are listed below:

\section{Fiscal Affairs Department (FAD):}

- June 2004: help the authorities move to a VAT, rationalize the import tariff, and overhaul the domestic indirect tax system.

- $\quad$ October 2004: review tax administration, including VAT implementation and help assess tax exemptions and incentives.

- $\quad$ September 2005: assess tax exemptions and incentives.

- June 2008: review and rationalize tax exemptions.

\section{Statistics Department (STA):}

- $\quad$ November 2003, January 2006: National accounts.

- February 2004: Balance of payments statistics.

- $\quad$ March 2004, April 2006, February 2007, August 2008: Government finance statistics.

- June 2004, May 2006, October 2007: Price statistics. 
- $\quad$ March 2007: Monetary statistics and reporting.

\section{Monetary and Capital Markets (MCM)}

- April 2004: Accounting, financial sector regulation, monetary operations and liquidity management.

- $\quad$ November 2005, March 2006, June 2006, November 2006, July 2007: Banking supervision, liquidity management, exchange regime and reserves management.

- March 2008: Macro-prudential indicators.

- $\quad$ November 2008, January 2009, March 2009: Financial Sector Assessment Program (FSAP), and a various follow up missions on banking supervision.

- $\quad$ February 2009, September 2009: Joint IMF-World Bank on debt management (DeMPA) and on Medium-Term Debt Strategy (MTDS).

- $\quad$ December 2010: Liquidity management and monetary policy operations.

\section{Legal (LEG):}

- $\quad$ October 2006, March 2008: Tax legislation.

- $\quad$ March 2007: AML/CFT initial assessment, and legal drafting on March 2008.

XIII. Resident Representative: None. 
Annex II. Cape Verde-Joint IMF-World Bank Management ACtion PlanIMPLEMENTATION MATRIX

\begin{tabular}{|c|c|c|c|}
\hline Title & Products & $\begin{array}{l}\text { Provisional Timing of } \\
\text { Missions }\end{array}$ & $\begin{array}{l}\text { Expected Delivery } \\
\text { Date }\end{array}$ \\
\hline \multicolumn{4}{|c|}{ A. Mutual information on relevant work programs } \\
\hline $\begin{array}{l}\text { Bank work program in } \\
\text { the next } 12 \text { months }\end{array}$ & $\begin{array}{l}\text { - PRSC-7 (IDA) } \\
\text { - Energy Operation } \\
\text { (IBRD) } \\
\text { - Country Economic } \\
\text { Memorandum } \\
\text { - PPIAF/TA on the energy } \\
\text { sector (develop } \\
\text { strategic/recovery plan for } \\
\text { Electra; Electra } \\
\text { management model) } \\
\text { - General economic } \\
\text { monitoring } \\
\text { - Transport assessment } \\
\text { (TACV) } \\
\text { - Non-lending technical } \\
\text { assistance } \\
\text { (public investment } \\
\text { management) } \\
\text { - Trust-Fund (Monitoring } \\
\text { \& Evaluation) }\end{array}$ & $\begin{array}{l}\text { August } 2011 \\
\text { July/Sept } 11 \\
\text { Sep } 2011 \\
\text { October } 2011 \\
\text { (ongoing) } \\
\text { October } 2011 \\
\text { November } 2011 \\
\text { October } 2011 \\
\text { October, } 2011\end{array}$ & $\begin{array}{l}\text { Q4 FY12 } \\
\text { Q2 FY12 } \\
\text { Q4 FY12 } \\
\text { Q4 FY13 } \\
\text { Q4 FY12 }\end{array}$ \\
\hline
\end{tabular}


Cape Verde: Joint Management Action Plan-Implementation Matrix (concluded)

\begin{tabular}{|c|c|c|c|}
\hline Title & Products & $\begin{array}{c}\text { Provisional Timing of } \\
\text { Missions }\end{array}$ & $\begin{array}{c}\text { Expected Delivery } \\
\text { Date }\end{array}$ \\
\hline \multicolumn{4}{|c|}{ A. Mutual information on relevant work programs } \\
\hline $\begin{array}{l}\text { IMF work program in } \\
\text { next } 12 \text { months }\end{array}$ & PSI program, 2nd review & November 2011 & $\begin{array}{l}\text { Board meeting } \\
\text { January } 2012\end{array}$ \\
\hline \multicolumn{4}{|c|}{ B. Agreement on joint products and missions } \\
\hline \multirow[t]{2}{*}{$\begin{array}{l}\text { Joint products in } \\
\text { next } 12 \text { months }\end{array}$} & $\begin{array}{l}\text { JSAN (Joint Staff } \\
\text { Assessment of the } \\
\text { Poverty Reduction } \\
\text { Strategy) }\end{array}$ & & Q4 FY12 \\
\hline & $\begin{array}{l}\text { Technical assistance on } \\
\text { MTDS }\end{array}$ & July 2011 & Q2FY12 \\
\hline
\end{tabular}




\section{AnneX III. CAPE Verde-Statistics Issues}

1. Data provision has some shortcomings, but is broadly adequate for surveillance. There is a need for substantial improvements in the areas of national accounts, financial sector, and balance of payments. The country has participated in the GDDS since February 2004. Cape Verde's plans for improvements of its statistical system are posted on the Fund's DSBB; the metadata of the monetary and financial sector were updated in August 2007. The country has benefited from STA technical assistance under the GDDS project for Lusophone Africa.

2. The authorities are taking steps to strengthen statistics, but the statistical system still suffers from a shortage of financial and human resources. A comprehensive master plan has been developed under the direction of the National Statistical Institute (INE), which assesses the need for upgrading the agencies that constitute the statistical system and outlines steps to broaden and improve all areas of statistics.

\section{Real sector statistics}

3. Although some improvements have been made to the national accounts, significant weaknesses remain, including a lack of reliable demand-side GDP. The timeliness of the data has not been improved, with the INE releasing production and expenditure-based GDP data for 2005-07 in 2010 and providing production-based GDP estimates for 2008 to the AFR mission in September 2010. However, the lag is still lengthy by international standards. INE is receiving technical assistance from Brazil and Spain in developing business indicators and national accounts. On May 2011, INE signed a cooperation protocol with its Spanish counterpart to enhance national accounts and produce environmental statistics. INE plans to publish quarterly GDP data by 2012.

4. INE is currently working on a complete overhaul of the national accounts. It is updating the national accounts benchmark and base years from 1980 to 2002 and adopting the 1993 SNA. The objective is timely compilation of GDP by industry and expenditure categories at current and constant prices as well as institutional sector accounts.

5. INE will also introduce Tourism Satellite Accounts. These accounts will measure upstream linkages between the tourism sector and the rest of the economy and the import content of tourism exports.

\section{Full implementation of the $1993 S N A$ would require a substantial improvement in} source data collection, for which capacity is currently lacking. The 2006 STA mission found a critical need to improve the timeliness and accuracy of national accounts source data, in particular the business survey. The capacity of INE staff working on national accounts was found to be overstretched; they assess in detail and correct individual source data entries - something not undertaken in most countries by national accounts compilers. The statistical master plan will address many of these challenges. 
7. A revamped CPI with new methodology was launched in February 2008. The previous official CPI was based on weights dating back to 1989, four years before imports were liberalized. Import liberalization considerably changed consumption patterns. INE has had assistance from the National Statistics Institute of Portugal and benefited from STA CPI missions in May-June, 2006, October 2006, and October 2007, to support the introduction and dissemination of the revised CPI. The new index has new weights and an updated commodity basket.

8. INE publishes labor market statistics only on annual basis with long delays. Recently, it published annual unemployment data, which is comparable to EUROSTAT and AFRISTAT. INE recently published the 2010 census. There is no data on wages in different sectors.

\section{Government finance statistics}

9. Fiscal data have been improved. Benefiting from TA under the GDDS project for Lusophone Africa, the Government Finance Statistics (GFS) compilation system is being upgraded. Most deposits previously held with commercial banks have been consolidated at the central bank. SIGOF, the Integrated Online Budget Management System, has been expanded to cover all semiautonomous institutes and most municipalities by the end of 2007 . The authorities have started reporting GFS for publication in the GFS Yearbook.

10. However quality is a serious concern. The fiscal accounts are subject to statistical discrepancies, flows and stocks are not always consistent. Significant delay in donor reporting of project financing also affects the accuracy of fiscal data. Despite the recent revision of external debt data, significant weaknesses affect preparation of debt sustainability analyses, especially regarding the PPG debt of state owned enterprises. Multilateral debt statistics regularly differ from data received by creditors, and debt service projections cannot be reconciled with the debt stock.

\section{Monetary and financial statistics}

11. The quality of monetary and financial statistics is adequate, in terms of both accuracy and timeliness. An STA mission undertaken in March 2007 helped Banco de Cabo Verde (BCV) to finalize the standardized report forms (SRFs) for reporting monetary statistics to STA. SRF-based monetary data have been published in the IFS Supplement since June 2007. These data are fully aligned with the recommendations of the Monetary and Financial Statistics Manual. The integrated monetary database that meets STA, AFR, and BCV statistical needs is now in place. Currently, there are gaps in tracking the source and direction of changes in emigrant deposits, which cause difficulties in assessing their interest sensitivity and gauging the appropriateness of the monetary policy stance.

\section{External sector statistics}

12. The accuracy, periodicity, and timeliness of balance of payments statistics compiled by BCV have continued to improve. A greater use of surveys, combined with the International Transactions Reporting System implemented by the BCV, has permitted a significant expansion of 
data sources and statistical coverage, which to a large extent follow the recommendations of the 5th edition of the Balance of Payments Manual. However, gaps in the tracking of large external flowsnotably FDI, private sector trade credit and debt stock-remains. Dissemination of quarterly BOP data on the BCV website has been regular and in 2007 the BCV resumed regular and timely transmission of these data to STA for publication in International Financial Statistics and in the Balance of Payments Statistics Yearbook. 


\section{Cape Verde: Common Indicators Required for Surveillance}

(As of July 7, 2011)

\begin{tabular}{|c|c|c|c|c|c|}
\hline & $\begin{array}{c}\text { Date of } \\
\text { Latest } \\
\text { Observation }\end{array}$ & $\begin{array}{l}\text { Date } \\
\text { Received }\end{array}$ & $\begin{array}{l}\text { Frequency } \\
\text { of Data }^{7}\end{array}$ & $\begin{array}{l}\text { Frequency } \\
\text { of } \\
\text { Reporting }^{7}\end{array}$ & $\begin{array}{l}\text { Frequency } \\
\text { of } \\
\text { Publication }^{7}\end{array}$ \\
\hline Exchange Rates & 04/30/11 & 06/06/11 & $\mathrm{D}$ & $\mathrm{D}$ & $\mathrm{D}$ \\
\hline $\begin{array}{l}\text { International Reserve Assets } \\
\text { and Reserve Liabilities of the } \\
\text { Monetary Authorities }\end{array}$ & Apr. 2011 & Jun. 2011 & $\mathrm{D}$ & W & M \\
\hline Reserve/Base Money & Apr. 2011 & Jun. 2011 & $\mathrm{D}$ & W & M \\
\hline Broad Money & Apr. 2011 & Jun. 2011 & M & $M$ & $M$ \\
\hline Central Bank Balance Sheet & Apr. 2011 & Jun. 2011 & $\mathrm{D}$ & W & M \\
\hline $\begin{array}{l}\text { Consolidated Balance Sheet of } \\
\text { the Banking System }\end{array}$ & Apr. 2011 & Jun. 2011 & M & M & M \\
\hline Interest Rates ${ }^{2}$ & Mar. 2011 & May 2011 & M & $M$ & $M$ \\
\hline Consumer Price Index & Apr. 2011 & May 2011 & M & M & M \\
\hline $\begin{array}{l}\text { Revenue, Expenditure, Balance } \\
\text { and Composition of Financing }{ }^{3}- \\
\text { Central Government }\end{array}$ & Mar. 2011 & May 2011 & Q & Q & Q \\
\hline $\begin{array}{l}\text { Stocks of Central Government } \\
\text { and Central Government- } \\
\text { Guaranteed Debt }\end{array}$ & Mar. 2011 & May 2011 & Q & Q & $A$ \\
\hline $\begin{array}{l}\text { External Current Account } \\
\text { Balance }\end{array}$ & Mar. 2011 & May 2011 & Q & Q & Q \\
\hline $\begin{array}{l}\text { Exports and Imports of Goods } \\
\text { and Services }\end{array}$ & Apr. 2011 & May 2011 & Q & Q & Q \\
\hline GDP/GNP & 2008 & Sept. 2010 & $A$ & $A$ & $A$ \\
\hline Gross External Debt & Mar. 2011 & May 2011 & Q & $\mathrm{Q}$ & A \\
\hline $\begin{array}{l}\text { International Investment } \\
\text { Position }\end{array}$ & 2007 & Aug. 2008 & $A$ & A & A \\
\hline
\end{tabular}

${ }^{1}$ Includes reserve assets pledged or otherwise encumbered as well as net derivative positions.

${ }^{2}$ Both market-based and officially-determined, including discount rates, money market rates, rates on treasury bills, notes and bonds.

${ }^{3}$ Foreign, domestic bank, and domestic nonbank financing.

${ }^{4}$ The general government consists of the central government (budgetary funds, extra budgetary funds, and social security funds) and state and local governments.

${ }^{5}$ Including currency and maturity composition.

${ }^{6}$ Includes external gross financial asset and liability positions vis-à-vis nonresidents.

${ }^{7}$ Daily (D), weekly (W), monthly (M), quarterly (Q), annually (A), irregular (I); and not available (NA). 


\section{IMF Executive Board Completes First Review Under Policy Support Instrument for Cape Verde}

The Executive Board of the International Monetary Fund (IMF) has completed the first review under a 15-month Policy Support Instrument (PSI) for Cape Verde. The Executive Board's decision was taken on a lapse of time basis. ${ }^{1}$

The review focused on policies to guide the country's continued recovery from the global economic crisis and to rebuild fiscal and reserve buffers. Economic recovery in Cape Verde continues unabated, driven by the tourism sector and a rapid implementation of the public investment program. Authorities remain committed to macroeconomic stability.

Cape Verde's PSI was approved on November 22, 2010 (see Press Release No. 10/457). The program's main objective is to enhance the sustainability of growth and development by maintaining a stable macroeconomic environment and implementing structural reforms. The IMF's framework for PSIs is designed for low-income countries (and small island states) that may not need IMF financial assistance, but still seek close cooperation with the IMF in preparation and endorsement of their policy frameworks.

\footnotetext{
${ }^{1}$ The Executive Board takes decisions under its lapse of time procedure when it is agreed by the Board that a proposal can be considered without convening formal discussions.
} 